\title{
MAGIC long-term study of the distant TeV blazar PKS 1424+240 in a multiwavelength context ${ }^{\star}$
}

J. Aleksić ${ }^{1}$, S. Ansoldi ${ }^{2}$, L. A. Antonelli ${ }^{3}$, P. Antoranz ${ }^{4}$, A. Babic ${ }^{5}$, P. Bangale ${ }^{6}$, U. Barres de Almeida ${ }^{6}$, J. A. Barrio ${ }^{7}$, J. Becerra González ${ }^{8,25, \star \star}$, W. Bednarek ${ }^{9}$, K. Berger ${ }^{8}$, E. Bernardini ${ }^{10}$, A. Biland ${ }^{11}$, O. Blanch ${ }^{1}$, R. K. Bock ${ }^{6}$, S. Bonnefoy ${ }^{7}$, G. Bonnoli ${ }^{3}$, F. Borracci ${ }^{6}$, T. Bretz ${ }^{12,26}$, E. Carmona ${ }^{13}$, A. Carosi ${ }^{3}$, D. Carreto Fidalgo ${ }^{12}$, P. Colin ${ }^{6}$, E. Colombo ${ }^{8}$, J. L. Contreras ${ }^{7}$, J. Cortina ${ }^{1}$, S. Covino ${ }^{3}$, P. Da Vela ${ }^{4}$, F. Dazzi ${ }^{6}$, A. De Angelis ${ }^{2}$, G. De Caneva ${ }^{10}$, B. De Lotto ${ }^{2}$, C. Delgado Mendez ${ }^{13}$, M. Doert ${ }^{14}$, A. Domínguez ${ }^{15,27}$, D. Dominis Prester ${ }^{5}$, D. Dorner ${ }^{12}$, M. Doro ${ }^{16}$, S. Einecke ${ }^{14}$, D. Eisenacher ${ }^{12}$, D. Elsaesser ${ }^{12}$, E. Farina ${ }^{17}$, D. Ferenc ${ }^{5}$, M. V. Fonseca ${ }^{7}$, L. Font ${ }^{18}$, K. Frantzen ${ }^{14}$, C. Fruck $^{6}$, R. J. García López ${ }^{8}$, M. Garczarczyk ${ }^{10}$, D. Garrido Terrats ${ }^{18}$, M. Gaug ${ }^{18}$, G. Giavitto ${ }^{1}$, N. Godinović ${ }^{5}$, A. González Muñoz ${ }^{1}$, S. R. Gozzini ${ }^{10}$, D. Hadasch ${ }^{19}$, M. Hayashida ${ }^{20}$, A. Herrero ${ }^{8}$, D. Hildebrand ${ }^{11}$, J. Hose ${ }^{6}$, D. Hrupec ${ }^{5}$, W. Idec $^{9}$, V. Kadenius ${ }^{21}$, H. Kellermann 6 , K. Kodani ${ }^{20}$, Y. Konno ${ }^{20}$, J. Krause ${ }^{6}$, H. Kubo ${ }^{20}$, J. Kushida ${ }^{20}$, A. La Barbera ${ }^{3}$, D. Lelas ${ }^{5}$, N. Lewandowska ${ }^{12}$, E. Lindfors ${ }^{21,28, \star \star}$, S. Lombardi ${ }^{3}$, M. López ${ }^{7}$, R. López-Coto ${ }^{1}$,

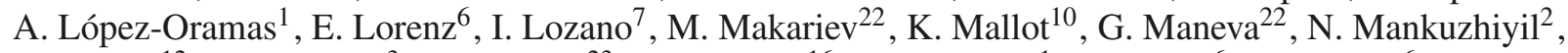
K. Mannheim ${ }^{12}$, L. Maraschi ${ }^{3}$, B. Marcote ${ }^{23}$, M. Mariotti ${ }^{16}$, M. Martínez ${ }^{1}$, D. Mazin ${ }^{6}$, U. Menzel ${ }^{6}$, M. Meucci ${ }^{4}$, J. M. Miranda ${ }^{4}$, R. Mirzoyan ${ }^{6}$, A. Moralejo ${ }^{1}$, P. Munar-Adrover ${ }^{23}$, D. Nakajima ${ }^{20}$, A. Niedzwiecki ${ }^{9}$, K. Nilsson $^{21,28}$, K. Nishijima ${ }^{20}$, N. Nowak ${ }^{6}$, R. Orito ${ }^{20}$, A. Overkemping ${ }^{14}$, S. Paiano ${ }^{16}$, M. Palatiello ${ }^{2}$, D. Paneque ${ }^{6}$, R. Paoletti ${ }^{4}$, J. M. Paredes ${ }^{23}$, X. Paredes-Fortuny ${ }^{23}$, S. Partini ${ }^{4}$, M. Persic ${ }^{2,29}$, F. Prada ${ }^{15,30}$, P. G. Prada Moroni ${ }^{24}$, E. Prandini ${ }^{11, \star \star}$,

S. Preziuso ${ }^{4}$, I. Puljak ${ }^{5}$, R. Reinthal ${ }^{21}$, W. Rhode ${ }^{14}$, M. Ribó ${ }^{23}$, J. Rico ${ }^{1}$, J. Rodriguez Garcia ${ }^{6}$, S. Rügamer ${ }^{12}$, A. Saggion ${ }^{16}$, T. Saito ${ }^{20}$, K. Saito ${ }^{20}$, M. Salvati ${ }^{3}$, K. Satalecka ${ }^{7}$, V. Scalzotto ${ }^{16}$, V. Scapin ${ }^{7}$, C. Schultz ${ }^{16}$, T. Schweizer ${ }^{6}$, S. N. Shore ${ }^{24}$, A. Sillanpää ${ }^{21}$, J. Sitarek ${ }^{1}$, I. Snidaric ${ }^{5}$, D. Sobczynska ${ }^{9}$, F. Spanier ${ }^{12}$, V. Stamatescu ${ }^{1}$, A. Stamerra ${ }^{3, \star \star}$,

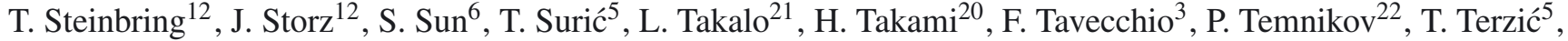

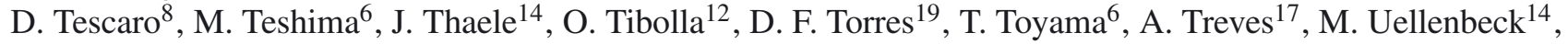
P. Vogler ${ }^{11}$, R. M. Wagner ${ }^{6,31}$, F. Zandanel ${ }^{15,32}$, R. Zanin ${ }^{23}$, (the MAGIC collaboration), S. Cutini ${ }^{3}$, D. Gasparrini ${ }^{3}$, A. Furniss ${ }^{33}$, T. Hovatta ${ }^{34}$, T. Kangas 28 , E. Kankare ${ }^{28}$, J. Kotilainen ${ }^{28}$, M. Lister ${ }^{35}$, A. Lähteenmäki ${ }^{36,37}$, W. Max-Moerbeck ${ }^{34}$, V. Pavlidou ${ }^{38}$, A. Readhead ${ }^{34}$, and J. Richards ${ }^{35}$

(Affiliations can be found after the references)

Received 1 Janaury 2014 / Accepted 29 April 2014

\section{ABSTRACT}

Aims. We present a study of the very high-energy (VHE; $E>100 \mathrm{GeV}$ ) $\gamma$-ray emission of the blazar PKS $1424+240$ observed with the MAGIC telescopes. The primary aim of this paper is the multiwavelength spectral characterization and modeling of this blazar, which is made particularly interesting by the recent discovery of a lower limit of its redshift of $z \geq 0.6$ and makes it a promising candidate to be the most distant VHE source. Methods. The source has been observed with the MAGIC telescopes in VHE $\gamma$ rays for a total observation time of $\sim 33.6 \mathrm{~h}$ from 2009 to 2011. A detailed analysis of its $\gamma$-ray spectrum and time evolution has been carried out. Moreover, we have collected and analyzed simultaneous and quasi-simultaneous multiwavelength data.

Results. The source was marginally detected in VHE $\gamma$ rays during 2009 and 2010, and later, the detection was confirmed during an optical outburst in 2011. The combined significance of the stacked sample is $\sim 7.2 \sigma$. The differential spectra measured during the different campaigns can be described by steep power laws with the indices ranging from $3.5 \pm 1.2$ to $5.0 \pm 1.7$. The MAGIC spectra corrected for the absorption due to the extragalactic background light connect smoothly, within systematic errors, with the mean spectrum in 2009-2011 observed at lower energies by the Fermi-LAT. The absorption-corrected MAGIC spectrum is flat with no apparent turn down up to $400 \mathrm{GeV}$. The multiwavelength light curve shows increasing flux in radio and optical bands that could point to a common origin from the same region of the jet. The large separation between the two peaks of the constructed non-simultaneous spectral energy distribution also requires an extremely high Doppler factor if an one zone synchrotron self-Compton model is applied. We find that a two-component synchrotron self-Compton model describes the spectral energy distribution of the source well, if the source is located at $z \sim 0.6$.

Key words. gamma rays: galaxies - BL Lacertae objects: individual: PKS 1424+240

\footnotetext{
* Appendix is available in electronic form at http://www. aanda.org

$\star \star$ Corresponding authors: J. Becerra Gonzalez, e-mail: jbecerra@iac.es; E. Lindfors, e-mail: elilin@utu.fi; E. Prandini, e-mail: elisa.prandini@unige.ch; A. Stamerra, e-mail: antonio.stamerra@i.infn.it
} 


\section{Introduction}

Blazars are active galactic nuclei (AGN) that host a relativistic jet, which is pointed at a small angle to the line of sight. The spectral energy distribution (SED) of blazars shows a two-bump structure. It is widely accepted that the lower energy bump is due to synchrotron emission produced by the relativistic electrons spiraling in the magnetic field of the jet. The location of the peak of this lower energy bump in the SED is used to classify the sources as low, intermediate, and high-synchrotron-peaked blazars (Abdo et al. 2010b).

The high energy-peaking blazars are the most numerous, extragalactic very high-energy (VHE, $E>100 \mathrm{GeV}$ ) $\gamma$-ray sources. The origin of the VHE $\gamma$-ray emission is still under debate. It is typically modeled with synchrotron selfCompton (SSC) emission models where the synchrotron radiation serves as seed photons for inverse Compton scattering (Maraschi et al. 1992). However, hadronic processes, such as proton synchrotron and radiation produced by the secondary particles, can also produce the observed VHE $\gamma$-ray emission (e.g., Mannheim \& Biermann 1992; Aharonian 2000).

Both models fit the observed IR, optical, X-ray, and $\gamma$-ray data well (e.g., Tavecchio et al. 2010; Reimer 2012), while it is generally assumed that the emission region is still opaque in radio bands and that the radio emission originates from a different emission region (Katarzynski et al. 2001).

The object PKS 1424+240 was discovered in the 1970s as a radio source (Fanti et al. 1974) and was identified as a blazar by Impey \& Tapia (1988). The source was detected in $\gamma$ rays by the Fermi Large Area Telescope (LAT; Atwood et al. 2009) with a very hard spectrum with a photon index of $\Gamma=1.85 \pm 0.07$ (Abdo et al. 2009), and it entered the family of VHE $\gamma$-ray emitters in spring 2009 when a detection was first reported by VERITAS (Ong 2009) and soon after confirmed by MAGIC (Teshima 2009). The source was previously observed during 2006 and 2007 by the MAGIC-I telescope, and a flux upper limit of $8.2 \%$ Crab Units $^{1}$ for $E>120 \mathrm{GeV}$ was derived (Aleksić et al. 2011). The VERITAS observations in 2009 indicated a steady flux with the photon spectrum, which is well described by a power law with a photon index of $3.8 \pm 0.5_{\text {stat }} \pm 0.3_{\text {syst }}$ and a flux normalization at $200 \mathrm{GeV}$ of $\left(5.1 \pm 0.9_{\text {stat }} \pm 0.5_{\text {syst }}\right) \times 10^{-11} \mathrm{TeV}^{-1} \mathrm{~cm}^{-2} \mathrm{~s}^{-1}$ (Acciari et al. 2010). As this manuscript was being resubmitted to the journal, the VERITAS collaboration reported observations of PKS1424+240 from 2009, 2011 and 2013, confirming the soft spectrum that had been reported with the 2009 dataset but indicated significant gamma-ray flux variability (Archambault et al. 2014) this time.

The source PKS $1424+240$ is a BL Lac object, which by definition shows weak or no emission lines in its optical spectra. Therefore, like for many BL Lacs, the redshift of PKS 1424+240 is still uncertain. Rau et al. (2012) reported a photometric upper limit of $z_{\mathrm{ul}}=1.1$. Recently Furniss et al. (2013) determined a lower limit of the redshift $z \geq 0.6035$ from the $\operatorname{Ly} \beta$ and $\operatorname{Ly} \gamma$ absorption. This is more distant than 3C $279(z=0.536)$, which was long considered to be the most distant VHE $\gamma$-ray emitter. Even though there are other sources with high lower limits on the redshift ${ }^{2}$, this makes PKS $1424+240$ a strong candidate to be the most distant known VHE gamma-ray emitter. Later, we

\footnotetext{
1 The Crab unit used in this work is an arbitrary unit obtained by dividing the integral energy flux measured above a certain threshold by the Crab nebula flux, which was measured above the same threshold by MAGIC (Albert et al. 2008a).

2 E.g. KUV 00311-1938, $z>0.506$ (Pita et al. 2012).
}

discuss the redshift constraints obtained from VHE $\gamma$-ray data analysis, which seem to confirm this result.

It should be noted that Meisner \& Romani (2010) report a detection of the host galaxy in the $i$-band, and assuming that the galaxies hosting BL Lac objects can be considered as standard candles (Sbarufatti et al. 2005), they estimated the redshift to be $z=0.23_{-0.05}^{+0.06}$. However, this value is in conflict with a new photometric lower limit from Shaw et al. (2013) and with the spectroscopic limit reported in Furniss et al. (2013).

In this paper, we present MAGIC observations of the source that include the first detection of the source in 2009, follow-up observations in 2010, and target of opportunity observations triggered by the optical high state of the source in Spring 2011. The differential and integral energy spectra are presented with a study of the spectral variability. We carried out an extensive multiwavelength study which makes use of the data available from $\gamma$ rays to radio to study multiwavelength properties and model the SED of the source.

\section{MAGIC observations and results}

\subsection{MAGIC data}

MAGIC (Major Atmospheric Gamma-ray Imaging Cherenkov) is a system of two $17 \mathrm{~m}$ Imaging Atmospheric Cherenkov Telescopes (IACT) located at the Roque de los Muchachos, Canary Island of La Palma at the height of $2200 \mathrm{~m}$ above sea level. The commissioning of the second MAGIC telescope finished at the end of 2009, and since then, both telescopes have worked together in stereoscopic mode (Cortina et al. 2009; Aleksić et al. 2012a). In simple terms, MAGIC observes the faint Cherenkov light emitted in the atmosphere by a shower of particles that is induced by a VHE $\gamma$ ray when entering the atmosphere. The light is focused into a camera and forms an image, which is triggered, registered, calibrated, and then parametrized with the so-called Hillas parameters (Hillas 1985). These parameters are used to separate the $\gamma$-like events, which constitute the signal, from the background dominated by hadronic events. In addition, dedicated Monte Carlo simulations of the system performance are used for the energy reconstruction and the $\gamma$-hadron separation.

The source PKS 1424+240 was observed in single-telescope mode (i.e. using only MAGIC-I) from April to June 2009, when MAGIC II was in commissioning phase, and observed in stereo mode in Spring 2010 and Spring 2011. Each data sample was analyzed independently since the performance of the instrument changed over the years.

The source PKS 1424+240 was observed in good conditions for $12.5 \mathrm{~h}$ in 2009 (from MJD 54938 to MJD 55 005), covering a zenith angle range between $4^{\circ}$ and $36^{\circ}$. In addition, stereo data were collected at the same zenith angle range in early 2010 between March to April (from MJD 55269 to MJD 55305) for a total observation time of $\sim 11.6 \mathrm{~h}$ and $9.5 \mathrm{~h}$ from April to May 2011 (from MJD 55875 to MJD 55 889). The whole data sample was taken in the false-source tracking (wobble) mode (Fomin et al. 1994), in which the telescopes were alternated every 20 min between two symmetric sky positions at $0.4^{\circ}$ offset from the source. The wobble enables us to take both source and background data simultaneously.

The analysis of the data was performed using the standard MAGIC analysis and reconstruction software (Albert et al. 2008a,b,c; Aliu et al. 2009). In the analysis of stereo data presented in this work, we took advantage of a direction reconstruction method based on the DISP RF method, as described 
Table 1. PKS 1424+240 observation characteristics and signal search results.

\begin{tabular}{ccccc}
\hline \hline Year & Obs. time & $E_{\text {th }}$ & $N_{\text {exc }}$ & Significance \\
\hline 2009 & $12.5 \mathrm{~h}$ & $\sim 150 \mathrm{GeV}$ & 498 & $4.6 \sigma$ \\
2010 & $11.6 \mathrm{~h}$ & $\sim 100 \mathrm{GeV}$ & 330 & $4.8 \sigma$ \\
2011 & $9.5 \mathrm{~h}$ & $\sim 100 \mathrm{GeV}$ & 333 & $5.5 \sigma$ \\
\hline
\end{tabular}

Notes. The year of observation, the observation time of the final selected sample, the energy threshold $\left(E_{\mathrm{th}}\right)$, the number of excess events $\left(N_{\text {exc }}\right)$, and the significance of the signal are reported.

in Aleksić et al. (2010), and adapted to the stereo observations (see Aleksić et al. 2012a for more details).

\subsection{Signal search}

The signal search is performed by making use of the distribution of the $\theta^{2}$ parameter, which is defined as the square of the angular distance between the reconstructed shower direction in the telescope camera and the real position of the source. To avoid systematic effects in the background calculation, we considered only events with the parameter size $e^{3}$ that is larger than 200 photo-electrons (mono analysis) and 50 photoelectrons (stereo analysis). The corresponding energy thresholds (defined as the energies where the number of Monte Carlo $\gamma$-ray events with assumed spectral indices is maximized in the histogram of the image size) are $\sim 150 \mathrm{GeV}$ for 2009 mono observations and $\sim 100 \mathrm{GeV}$ for 2010 and 2011 stereoscopic observations.

The signal is extracted by comparing the $\theta^{2}$ distribution of the source region $(\mathrm{ON})$ with the background $(\mathrm{OFF})$, which is estimated from one (in 2010 and 2011 data samples) and three regions (in the 2009 data sample) of the sky located near the source of interest and collected simultaneously with the source observation. The significance of the detection for the different data samples can be found in Table 1. During the 2009 observational campaign, a hint of a signal was found with a number of excess events $N_{\mathrm{ex}} \approx 498\left(N_{\mathrm{ex}}=N_{\mathrm{ON}}-N_{\mathrm{OFF}}\right)$ and $4.6 \sigma$, which are calculated according to Eq. (17) of Li \& Ma (1983). This hint of signal was confirmed later in the 2010 and 2011 campaigns: the $\theta^{2}$ distribution from the 2010 sample gives $\sim 330$ excess events with a significance of $4.8 \sigma$, while an excess of $N_{\text {ex }} \sim 333$ was found corresponding to a significance of $5.5 \sigma$ in the 2011 sample. The stacked significance of the overall sample $(33.6 \mathrm{~h}$ of data from 2009 to 2011,1161 excess events) is more than $7 \sigma$.

\subsection{Differential energy spectra}

The differential energy spectra observed with the MAGIC telescopes in 2009, 2010, and 2011 campaigns are shown in Fig. 1. In each case, the spectrum can be well fit with a simple power law of the form:

$$
\frac{\mathrm{d} N}{\mathrm{~d} E}=f_{0} \cdot\left(\frac{E}{200 \mathrm{GeV}}\right)^{-\Gamma} \quad\left[\frac{\mathrm{ph}}{\mathrm{cm}^{2} \mathrm{~s} \mathrm{TeV}}\right] .
$$

The parameters resulting from the fits are compatible within the uncertainties and are reported in Table 2. The systematic uncertainties in the spectral measurements with MAGIC for spectra similar to that of the Crab nebula (i.e. bright and relatively hard spectra) were reported in Albert et al. (2008d)

\footnotetext{
3 The parameter size represents the total number of photo-electrons in an image.
}

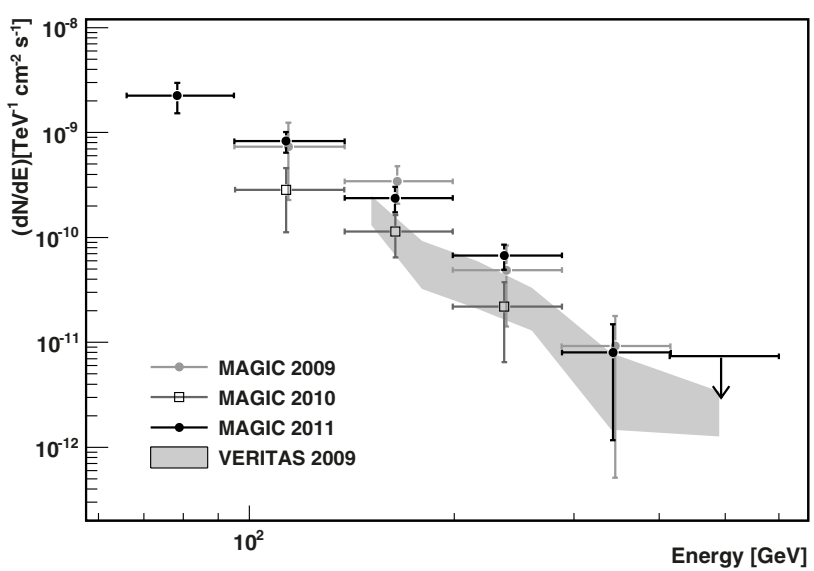

Fig. 1. Differential energy spectra of PKS 1424+240 measured by MAGIC in 2009 (gray circles), 2010 (squares), and 2011 (black circles). The black downward arrow represents the $95 \%$ confidence level upper limit for 2011 data. The gray-shaded area represents the results obtained in 2009 with VERITAS (Acciari et al. 2010).

and Aleksić et al. (2012a) for mono and stereo observations, which were about $11 \%$ in the normalization factor (at $300 \mathrm{GeV}$ ) and $0.15-0.20$ in the photon index. However, for spectral shapes described by a photon index of 4 and gamma-ray fluxes lower than that of the Crab by one order of magnitude, the systematic errors increase substantially. Considering the spectral shape of PKS $1424+240$, the relatively low dynamic range over which MAGIC detects photons (100-400 GeV), and the relatively low signal-to-noise background of the different datasets, we estimated the systematic uncertainties to be $30 \%$ in the flux level and 0.7 in the power-law index for the 2009 dataset (obtained with mono observations), $20 \%$ and 0.54 for the flux and the power-law index for the 2010 dataset (obtained with stereo observations) respectively, and $19 \%$ and 0.22 for the flux and the power-law index for the 2011 dataset (obtained with stereo observations, where the gamma-ray flux is about twice that measured in 2010), respectively. The error on the flux does not include uncertainty on the energy scale. The energy scale of the MAGIC telescopes is determined with a precision of about $17 \%$ at low energies $(E<100 \mathrm{GeV})$ and $15 \%$ at medium energies $(E<300 \mathrm{GeV})$ (Aleksić et al. 2012a).

The mean differential flux registered by VERITAS between February to June 2009 is significantly lower from the spectrum observed by MAGIC I in the same period. This apparent discrepancy is likely related to the different time coverage of the two observations and is discussed later. The spectral slope we derive is consistent with the results presented in Acciari et al. (2010). However, unlike the method in Acciari et al. (2010), we do not detect excess events above $400 \mathrm{GeV}$ in any data sample. We therefore derive a $95 \%$ confidence level upper limit for the energy bin $416-601 \mathrm{GeV}$ of $7.2 \times 10^{-12} \mathrm{TeV}^{-1} \mathrm{~cm}^{-2} \mathrm{~s}^{-1}$ for 2011 data. This limit agrees with the value measured by Acciari et al. (2010) at $\sim 500 \mathrm{GeV}$.

\subsection{VHE variability analysis}

Table 2 reports the integral fluxes measured by MAGIC from 2009 to 2011 above $150 \mathrm{GeV}$, which are drawn in Fig. 2 (dashed lines). All the fluxes are below the upper limit derived from the previous MAGIC observation in 2006 and 2007, which was (above $120 \mathrm{GeV}$ at $95 \%$ confidence level) $3.1 \times$ $10^{-11} \mathrm{~cm}^{-2} \mathrm{~s}^{-1}$ (Aleksić et al. 2011). 
Table 2. PKS 1424+240 spectrum power-law fit parameters and integral flux values.

\begin{tabular}{ccccc}
\hline \hline Period & $\begin{array}{c}\text { Fit range } \\
{[\mathrm{GeV}]}\end{array}$ & $\begin{array}{c}f_{0} \\
{\left[\mathrm{~cm}^{-2} \mathrm{~s}^{-1} \mathrm{TeV}^{-1}\right]}\end{array}$ & $\Gamma$ & $\begin{array}{c}F(>150 \mathrm{GeV}) \\
{\left[\mathrm{cm}^{-2} \mathrm{~s}^{-1}\right]}\end{array}$ \\
\hline 2009 & $150-400$ & $\left(1.3 \pm 0.6_{\text {stat }} \pm 0.4_{\text {sys }}\right) \times 10^{-10}$ & $5.0 \pm 1.7_{\text {stat }} \pm 0.7_{\text {sys }}$ & $(1.66 \pm 0.50) \times 10^{-11}$ \\
2010 & $100-300$ & $\left(0.5 \pm 0.2_{\text {stat }} \pm 0.1_{\text {sys }}\right) \times 10^{-10}$ & $3.5 \pm 1.2_{\text {stat }} \pm 0.5_{\text {sys }}$ & $(0.53 \pm 0.25) \times 10^{-11}$ \\
2011 & $100-400$ & $\left(1.0 \pm 0.3_{\text {stat }} \pm 0.2_{\text {sys }}\right) \times 10^{-10}$ & $3.9 \pm 0.7_{\text {stat }} \pm 0.2_{\text {sys }}$ & $(1.00 \pm 0.30) \times 10^{-11}$ \\
\hline
\end{tabular}

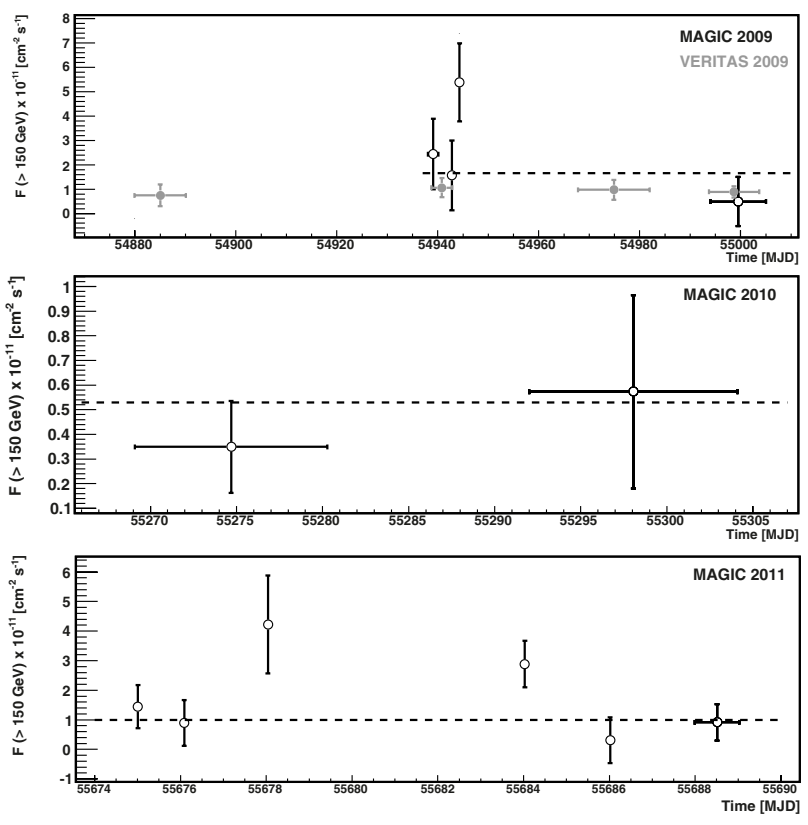

Fig. 2. VHE $\gamma$-ray light curve of PKS $1424+240$ for $E>150 \mathrm{GeV}$ from the observation campaigns in 2009, 2010 and 2011 with the MAGIC telescopes. Dashed lines represent the mean MAGIC fluxes, while the gray points represent the light curve measured by VERITAS in 2009, as adapted from Acciari et al. (2010).

A constant flux hypothesis is consistent with the yearly values from 2009 to 2011 at $95 \%$ confidence level $\left(\chi^{2} /\right.$ d.o.f. $\left.=4.4 / 2\right)$. In addition, constant fits to the light curves of the individual years also agree with the hypothesis of constant flux at the same confidence level.

Interestingly, VERITAS found no variability in the 2009 dataset in the energy range of 140-600 GeV. In Fig. 2, the gray markers refer to VERITAS 2009 observations from Acciari et al. (2010). Since VERITAS reported the integral flux in the range $140-600 \mathrm{GeV}$, the fluxes have been scaled down by $18 \%$ (derived from the spectral index of $\Gamma=3.8$ measured by VERITAS) to be compared to the MAGIC light curve at above $150 \mathrm{GeV}$. The statistical errors of the MAGIC flux measurements are larger than those from VERITAS because the MAGIC 2009 observations were performed with a single telescope $^{4}$ and were substantially shorter (12 h vs. $28 \mathrm{~h}$ ) than those from VERITAS.

\subsection{Redshift estimation from $\gamma$-ray observations}

The redshift of PKS $1424+240$ is still uncertain and can be constrained by VHE $\gamma$-ray observations by considering the interaction of energetic photons with the diffuse optical and nearinfrared background, which are also called the extragalactic

\footnotetext{
4 The MAGIC Stereo sensitivity is a factor of 3 better than the one of the MAGIC I telescope at the lowest VHE energies (Aleksic et al. 2012a).
}

background light (EBL) (Hauser \& Dwek 2001). The interaction of VHE photons with the EBL leads to electron-positron pair production. The effect is a partial or total absorption of the VHE photons coming from distant sources and a consequent distortion of the emitted spectrum. Due to the large uncertainties in the direct EBL measurements, many models have been proposed in recent years (Stecker et al. 2006, 2012; Franceschini et al. 2008; Gilmore et al. 2009; Kneiske \& Dole 2010; Domínguez et al. 2011).

In Acciari et al. (2010), the authors present the photon index measured with Fermi-LAT at low energies in combination with VERITAS data corrected by the EBL absorption effect using three recent EBL models (Franceschini et al. 2008; Gilmore et al. 2009; Finke et al. 2010) and derive an upper limit of the redshift of $z<0.66$ with a $95 \%$ confidence level.

A similar approach is undertaken in Prandini et al. (2010, 2011), where the idea that the slope of the VHE spectrum corrected for EBL absorption should not be harder than the one measured by Fermi-LAT at lower energies is tested on a sample of known redshift sources. The redshifts $z^{*}$ at which the two slopes match are compared to the spectroscopic redshifts, $z_{\mathrm{spec}}$, with the result that $z^{*}$ is above $z_{\text {spec }}$ in all the cases considered. Therefore, $z^{*}$ can be used as upper limit estimate of the source distance if there is no spectroscopic redshift available.

If we apply the same method to the data presented here, and in particular, to the 2011 spectral points that are the most precise, we obtain $z^{*}=0.61 \pm 0.10$, where $z^{*}$ is the redshift at which the EBL-deabsorbed spectrum has the same slope as measured by Fermi-LAT at lower energies, by assuming the Franceschini et al. (2008) EBL model. This implies a $2 \sigma$ upper limit on the redshift of 0.81 .

The upper limit derived agrees with the value obtained by Furniss et al. (2013), and, therefore, we hereafter adopt the redshift of $z=0.6$ for the source to not overestimate the EBL absorption.

\section{Multiwavelength view of PKS 1424+240}

The multiwavelength data for PKS 1424+240 were collected from several ongoing monitoring programs in radio, optical, $\mathrm{X}$-rays and high-energy $\gamma$ rays. The resulting multiwavelength light curves are shown in Fig. 3.

\subsection{Radio data}

The object PKS $1424+240$ is monitored at $15 \mathrm{GHz}$ using the $40 \mathrm{~m}$ telescope of the Owens Valley Radio Observatory as a part of a larger monitoring program, where a sample of $\sim 1700$ sources is observed twice a week (Richards et al. 2011). The telescope is equipped with dual-beamed, off-axis optics and a cooled receiver installed at the prime focus. The two sky beams are Dicke switched using the off-source beam as a reference, and the source is alternated between the two beams in an ON-ON fashion to remove atmospheric and ground contamination. The calibration reference source is 3C 286 for which the flux density of $3.44 \mathrm{Jy}$ at $15 \mathrm{GHz}$ is assumed (Baars et al. 1977). 


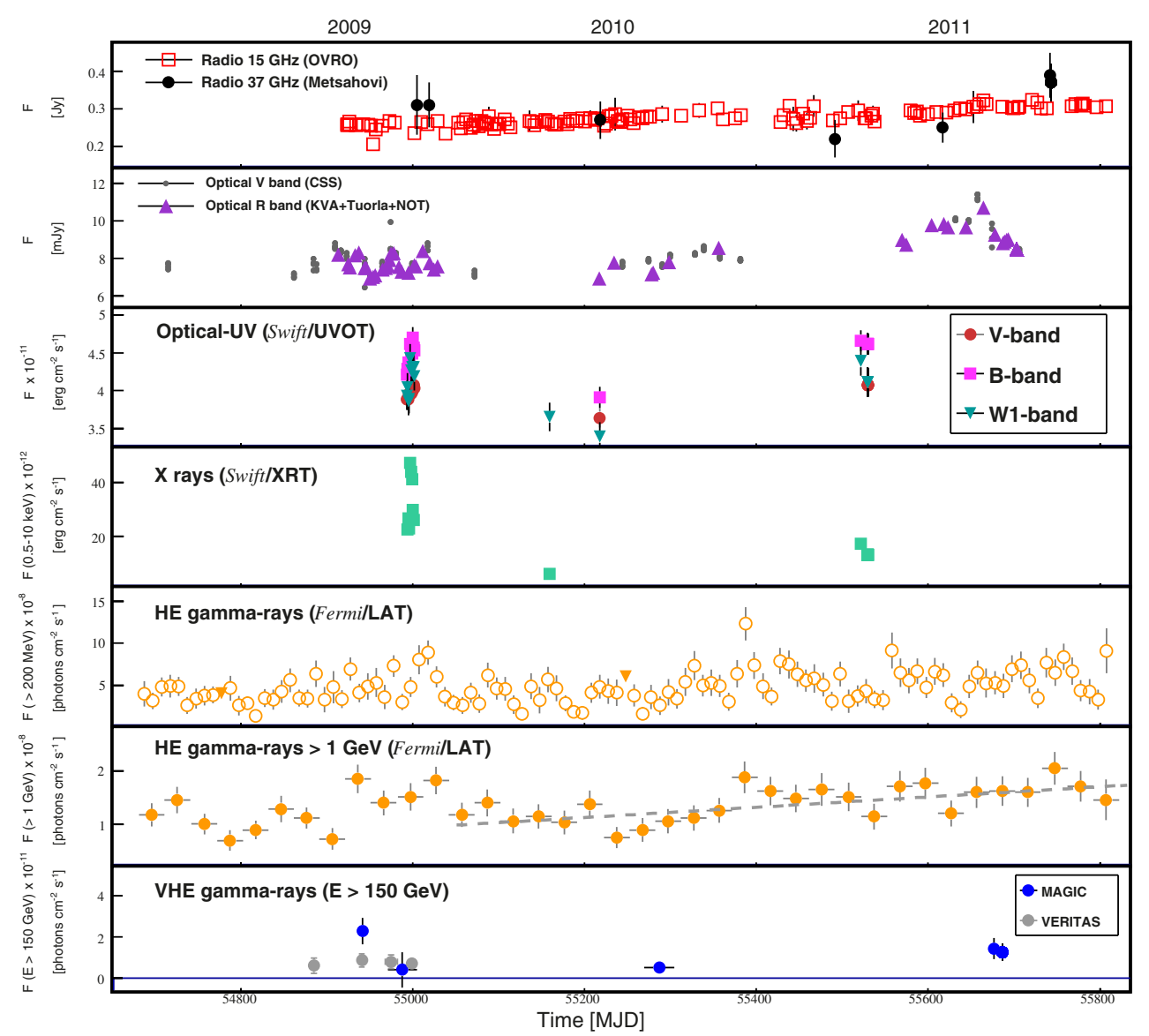

Fig. 3. Multiwavelength light curve of PKS 1424+240 from MJD 54700 to MJD 55800 . Since all Swift/UVOT filter data show the same trend, only filters $B, V$, and $W 1$ are shown. The dashed gray line represents a linear fit to the Fermi-LAT data after the X-ray flare.

The systematic uncertainty of about $5 \%$ in the flux density scale is not included in the error bars. Details of the observations, calibration, and analysis are given in Richards et al. (2011).

Visual inspection of the $15 \mathrm{GHz}$ light curve shows an increasing trend from 2009 to 2011; the average flux increases from $0.26 \pm 0.01 \mathrm{Jy}$ (MJD 54923) to $0.31 \pm 0.01 \mathrm{Jy}$ (MJD 55 807) with minimum and maximum values of $0.20 \pm$ $0.01 \mathrm{Jy}$ (MJD 54954) and $0.32 \pm 0.02 \mathrm{Jy}$ (MJD 55 721), respectively. The constant hypothesis can be discarded due to its low probability $\left(\chi^{2} /\right.$ d.o.f. $=861 / 126$, probability smaller than 0.0001).

We test the trend by using the five methods for obtaining the linear regression fits from Isobe et al. (1990) and find the trend to be significant at the $>20 \sigma$ level. We also calculate the intrinsic modulation index of the light curve using the likelihood method introduced in Richards et al. (2011). The intrinsic modulation index is a measure of variability, similar to the standard variability index (e.g. Aller et al. 1992) but takes the errors and sampling into account in the calculation of the likelihood (see Richards et al. 2011, for further details). For the radio data, we obtain a modulation index of $0.072 \pm 0.005$. This value confirms that the source is significantly variable and the variability amplitude is close to the mean value of $0.036 \pm 0.010$ obtained for a sample of 98 HSP BL Lacs in the OVRO sample (Richards et al. 2014).

The visual inspection also suggests a small amplitude flare in 2011. The existence of this flare was tested by removing the increasing trend by fitting a line to data and then calculating the intrinsic modulation index using the likelihood method. Because this method takes the flux density of the source into account, the average flux density $0.28 \mathrm{Jy}$ was added to the residuals.
The intrinsic modulation index for the de-trended data is then $0.026 \pm 0.003$, implying that the light curve is indeed variable above the $3 \sigma$ level even if the trend is removed.

The source has also been observed by the Metsähovi $13.7 \mathrm{~m}$ radio telescope at $37 \mathrm{GHz}$. The measurements were made with a $1 \mathrm{GHz}$-band dual beam receiver centered at $36.8 \mathrm{GHz}$. The observations are $\mathrm{ON}-\mathrm{ON}$ observations, which alternate the source and the sky in each feed horn. A typical integration time to obtain one flux density data point is between 1200 and 1400 s. A detailed description of the observation and analysis methods can be found in Teräsranta et al. (1998). The detection limit (defined as $S / N \geq 4$ ) of the telescope is on the order of 0.2 Jy under optimal weather conditions. Given that the typical flux density of PKS $1424+240$ at $37 \mathrm{GHz}$ is close to this limit, the source can usually be observed during good weather only, and therefore, the observed light curve from this source is sparse. Figure 3 (first panel from the top) shows the significant detections of the source from 2009 to 2011. The measured flux densities are between $0.2-0.35 \mathrm{Jy}$, which is close to the detection limit and results in large measurement errors. Within these error bars, the light curve does not show significant variability.

In addition to single dish observations, the source has also been observed with the Very Long Baseline Array (VLBA) at $15 \mathrm{GHz}$ as a part of the $\gamma$-ray selected MOJAVE sample (Lister et al. 2011). Analysis of all seven observed MOJAVE epochs up to now reveals two moving components with speeds of $55 \pm 24 \mu \mathrm{arcsec} / \mathrm{y}$ and $51 \pm 9 \mu \operatorname{arcsec} / \mathrm{y}$ (M. Lister, priv. comm.). Using $z=0.6$, this converts to a speed of $\sim 1.2 c$ and Doppler factor $\delta=5.5$ (assuming an average BL Lac object viewing angle of $5^{\circ}$ as determined by Hovatta et al. 2009). Note 
that a viewing angle of $1^{\circ}$ results in $\delta=12$, which is rather slow but is in accordance with high-energy peaking BL Lac objects showing lower Doppler factors in the $\gamma$-ray loud AGN (Lister et al. 2011). The VLBA core is resolved in the MOJAVE images and has a mean-fitted FWHM Gaussian of 0.1 milliarcsec, which corresponds to $0.67 \mathrm{pc}$ at $z=0.6$. The images were also investigated for limb brightening, which is considered to be a signature of a spine-sheath structure, and recent observations of Mrk 501, for example, have shown limb brightening (Piner et al. 2009). However, for PKS 1424+240 we find no signature of limb brightening.

Simultaneous single-dish and VLBA observations show that the extended jet contributes $\sim 60 \mathrm{mJy}$ to the total single dish $15 \mathrm{GHz}$ flux density (M. Lister, priv. comm.).

\subsection{Optical data}

The source has been observed as part of the Tuorla blazar monitoring program since 2006. The observations are done with the Kungliga Vetenskapsakademi (KVA) telescope on La Palma, which is operated remotely from Finland and the Tuorla $1 \mathrm{~m}$ telescope, located in Finland. KVA consists of two telescopes, the larger one being a $60 \mathrm{~cm}(f / 15)$ Cassegrain telescope equipped with a CCD polarimeter capable of polarimetric measurements in $B V R I$-bands that uses a plane-parallel calcite plate and a super-achromatic $\lambda / 2$ retarder. The second, a $35 \mathrm{~cm}$ Celestron telescope, can be used for photometric measurements in $B, V$, and $R$-bands. The observations of PKS $1424+240$ are done in the $R$-band and analyzed using the standard procedures with the pipeline developed for the monitoring program (Nilsson et al., in prep.). The magnitudes are measured with differential photometry by comparing star magnitudes from Fiorucci et al. (1996). The polarimetric measurements were done without filters to improve the signal-to-noise of the observations. The degree of polarization and position angle were calculated from the intensity ratios of the ordinary and extraordinary beams using standard formulae and semiautomatic software specially developed for polarization monitoring purposes.

In April 2011 during the high optical state of the source, observations were also performed at the Nordic Optical Telescope to get a better sampling of the light curve, as KVA was suffering from technical problems. The data were reduced using the same procedure as for KVA and Tuorla data.

The object PKS 1424+240 has also been observed as part of the Catalina Sky Survey (CSS) Program in the optical $V$-band. The data are publicly available (Drake et al. 2009) ${ }^{5}$ and are used to get better sampling for the optical light curve.

During the six years of monitoring, the optical $R$-band magnitude of the source was between 13.6 and 14.5 ( $V$-band: 13.9 and 14.6), and after the beginning of 2009, the source has been brighter than $R=14.2$ in all measurements ( $V$-band: 14.3). Historical data from 1994-1995 (Fiorucci et al. 1996) and 1988 (Mead et al. 1990) show $R$-band magnitudes from 14.75 (1988) to 14.2 (1995) and the $V$-band measurement from 1984 shows $V=16.2$. The optical magnitudes measured from 2009 to 2011, therefore, clearly present an optical high state of the source when compared to historical data. Within these three years of data, the average magnitude has been $R \sim 14$ (7.7 mJy) with the highest optical flux in 2011. The maximum flux reached $R=13.65(10.7 \mathrm{mJy})$, which is $\sim 40 \%$ above the average core flux from 2009-2011, and is the highest

\footnotetext{
5 http://nesssi.cacr.caltech.edu/DataRelease/
}

optical flux measured from the source to our knowledge. The 2011 MAGIC observations were triggered by this high optical flux. However, the data taking of MAGIC started when the optical flux was already decreasing and the average optical flux during 2011 MAGIC observations was $R=13.83$ (9.0 mJy), while it was $R=14.01$ (7.68 mJy) and $R=14.05(7.39 \mathrm{mJy})$ in 2009 and 2010, respectively.

The optical light curves in the $R$ - and $V$-bands start three years earlier than the $15 \mathrm{GHz}$ light curves, but the increasing trend seems to be present also in optical data. We also test for the existence of this trend in radio data. In the optical $R$-band, the trend is significant at the $19 \sigma$ level. The intrinsic modulation index of the light curve is $0.152_{-0.012}^{+0.010}$, and with the trend subtracted, it is $0.071 \pm 0.005$. The same analysis is repeated for the $V$-band data (which have better sampling in 2006-2008), resulting in a lower but still highly significant trend at the $9 \sigma$ level.

In 2011, we also performed two polarization measurements: May 6th (MJD 55688 ) and May 21st (MJD 55 703). The polarization was found to be $7.2 \pm 0.5 \%$ and $9.1 \pm 0.6 \%$, respectively. Compared to historical observations from 1984 and 1988 (without filter $4.7 \pm 0.3 \%$ (Impey \& Tapia 1990) and $R$-band $4.9 \pm$ $0.3 \%$ (Mead et al. 1990)), the polarization is significantly higher. The polarization degree is still within the typical range for BL Lacs (e.g. Jannuzi et al. 1993).

\subsection{Swift UV and X-ray observations}

Swift observations of PKS 1424+240 were performed using two of the three on-board instruments: The X-ray telescope (XRT, Burrows et al. 2005) covers the $0.2-10 \mathrm{keV}$ energy band, and the UV/Optical Telescope (UVOT, Roming et al. 2005) covers the 180-600 $\mathrm{nm}$ wavelength range with $V, B, U, U V W 1$, $U V M 2$, and $U V W 2$ filters. The third instrument, the Burst Alert Telescope (BAT, Barthelmy et al. 2005), is a coded-mask imager that covers the $15-150 \mathrm{keV}$ energy range. The source has not been detected by the complete analysis of 54 months of the BAT survey data, or the 2nd Palermo BAT catalog (Cusumano et al. 2010), and therefore, the BAT data were not used in the present analysis. In the following, the XRT data from 0.2 to $10 \mathrm{keV}$ are discussed with the UVOT data.

Swift observed the source extensively in June 2009, following the detection of VHE emission by VERITAS (Ong 2009; Acciari et al. 2010); a few sporadic observations followed in November 2009, January 2010, and November 2010, summing up to a total of nearly $26 \mathrm{ks}$ of observations. Table A.1 summarizes the Swift observations of PKS 1424+240.

The XRT data were processed using the FTOOLS task XRTPIPELINE (version 0.12.6), which is distributed by HEASARC within the HEASoft package (v6.10). Events with grades 0-12 were selected for the data (see Burrows et al. 2005) and response matrices version 20100802 available in the Swift CALDB were used. For the spectral analysis, the modest pileup affecting the June 2009 data was evaluated following the standard procedure ${ }^{6}$, which resulted in a piled-up region with a radius of $\sim 7$ arcsec. This region was masked, and the signal was extracted within an annulus with inner radius of 3 pixels ( $7.1 \mathrm{arcsec})$ and outer radius of 25 pixels $(59 \mathrm{arcsec})$. The pileup correction was applied only to observations with count rates higher than 0.6 cps. The observations with ID 00038104001 and 00038104010 show a strip of dead pixels crossing the source; although the xrtpipeline creates an ancillary response file (ARF) correcting for the exposure map, we excluded these

6 http://www.swift.ac.uk/analysis/xrt/pileup.php 
data from our analysis. The observations with ID 00039182002 and 00039182003 have few counts due to the low source flux and short observation time. The low statistics do not allow us to perform a reliable spectral fit, and for this reason, they have been excluded.

The spectra were extracted from the corresponding event files and binned using GRPPHA to ensure a minimum of 27 counts per bin in a manner, so that the $\chi^{2}$ statistic could be used reliably. Spectral analyses were performed using XSPEC version 12.6.0.

The results are summarized in Appendix A. Table A.2 shows the results of a power-law model fit to the data. Photo-electric absorption with a hydrogen column density fixed to the Galactic value $n_{\mathrm{H}}=3.1 \times 10^{20} \mathrm{~cm}^{-2}$ (Kaberla et al. 2005) was included in the model.

At the beginning of the observations, the source was in a high X-ray state compared to the lower X-ray states in 2010. A flare has been observed on June 15, 2009 lasting 3 days (Fig. 3) with the flux doubling on a one-day timescale. During the burst, a spectral hardening is recognizable, although a clear harder-when-brighter trend cannot be seen in the whole data set.

The observed day-scale X-ray variability sets an upper limit for the size of the $\mathrm{X}$-ray emission region given by the causality relation: $R<c t_{\mathrm{var}} \delta /(1+z) \sim 1.6 \times 10^{15} \delta[\mathrm{cm}]$ by assuming a redshift of 0.6 .

Swift/UVOT observed the source with all filters $(V, B, U$, $U V W 1, U V M 2, U V W 2$ ) over 12 days. During three of those days, the filter-of-the-day mode was used. The UVOT source counts were extracted from a 5 arcsec-sized circular region centered on the source position, while the background was extracted from a nearby larger, source-free, circular region. This data were processed with the uvotmaghist task of the HEASOFT package. The observed magnitudes have been corrected for Galactic extinction $E(B-V)=0.059$ mag (Schlegel et al. 1998), applying the formulas by Pei (1992) and finally converting them into fluxes following Poole et al. (2008). The observed magnitudes and the de-reddened fluxes are collected in Table A.3. The UV brightness is marginally variable, increasing during the X-ray flare of June 15.

\subsection{Fermi-LAT $\gamma$-ray data}

Fermi-LAT is a pair-conversion $\gamma$-ray telescope sensitive to photon energies greater than $20 \mathrm{MeV}$. In its nominal scanning mode, it surveys the whole sky every $3 \mathrm{~h}$ with a field of view of about 2.4 steradians (Atwood et al. 2009). The LAT data presented in this paper were collected from MJD 54682 (2008 August 4) to MJD 55200 (2011 June 4). An unbinned likelihood spectral analysis was performed to produce the light curve with the standard analysis tool gtlike, which included in the Science Tools software package (version v09r23p01) ${ }^{7}$. The P7_SOURCE_V6 instrument response functions were used, which is a refinement to previous LAT response functions, reflecting improved understanding of the point-spread function and effective area (Ackermann et al. 2012). For this analysis, only photons located in a circular region of interest (ROI) with a 10 degree radius, centered at the position of PKS1424+240, were selected. In addition, we excluded photons arriving from zenith angles $>100^{\circ}$ to limit contamination from Earth limb $\gamma$ rays, and events are detected while the

\footnotetext{
7 For a documentation of the Science Tools, see http://fermi. gsfc.nasa.gov/ssc/data/analysis/documentation/
}

spacecraft rocking angle was $>52^{\circ}$ to avoid time intervals during which Earth entered the LAT field of view (FoV).

For the $\gamma$-ray analysis, we used the Galactic and Isotropic diffuse emission models gal_2yearp7v6_v0.fits and iso_p7v6source.txt. The isotropic background is defined as the sum of residual cosmic-ray background and extragalactic diffuse $\gamma$-ray background (file provided with Science Tools) ${ }^{8}$. All point sources in the Fermi-LAT Second Source Catalog (2FGL; Nolan et al. 2012) are within $20^{\circ}$ of PKS1424+240, including the source of interest itself, were considered in the analysis. Those within the ROI were fitted with power-law models with spectral indices set to the values obtained from the likelihood analysis of the full data set, while those beyond $10^{\circ}$ radius ROI had their values frozen to those found in the 2FGL. Upper limits at 2-sigma confidence level were computed for time bins with test statistics (TS) ${ }^{9}<9$ and when the nominal flux uncertainty is larger than half the flux itself (Abdo et al. 2010a). All the uncertainties stated here are statistics only, the estimated systematic uncertainty of the integral fluxes above $200 \mathrm{MeV}$ and $1 \mathrm{GeV}$ are both about $10 \%$ for a hard source like PKS 1424+240 (Ackermann et al. 2012).

The spectral analysis was performed in the full band (from $100 \mathrm{MeV}$ to $300 \mathrm{GeV}$ ) for the whole 2008-2011 period using a simple power-law model. The best-fit parameters for the full-band model are $\Gamma=1.784 \pm 0.016$ and an integral flux of $(7.7 \pm 0.2) \times 10^{-8} \mathrm{ph} \mathrm{cm}^{-2} \mathrm{~s}^{-1}$ with the corresponding detection significance given by the $\sqrt{T S} \sim 86 \sigma$.

The Fermi-LAT integral flux light curves above $200 \mathrm{MeV}$ and $1 \mathrm{GeV}$ were derived by performing separate flux estimations with 10- and 30-day bins, respectively, and are shown together with the multiwavelength light curves. They show a flickering behavior, and no strong activity is detected during the investigated period. A constant flux hypothesis has a low probability $\left(\chi^{2} /\right.$ d.o.f. $=225 / 110$, incompatible with the hypothesis of constant flux at $95 \%$ confidence level). The light curves have a hint of a fast flare occurring at the same time as the X-ray flare in 2009 that was detected, and another similar event was observed in July 2010 (MJD 55 748), but no X-ray or VHE observations are available during this flare. No spectral variation is observed, even during the $\mathrm{X}$ ray flares. The fitted value of the spectral index is consistent with being constant in time with a probability value of $93 \%$, which agrees with the hypothesis that there is no significant spectral variations at a $95 \%$ confidence level.

\subsection{Multiwavelength light curve}

The radio light curve at $15 \mathrm{GHz}$ and the optical light curves $(R$ and $V$-bands) show a clear increase of flux with time, as concluded in Sects. 3.1 and 3.2. In the HE $\gamma$-ray light curves, the flux level seems to slightly increase toward the 2011 season. In particular, for Fermi-LAT data above $1 \mathrm{GeV}$ (after June 2009), the likelihood ratio test indicates a non-zero slope with more than $99 \%$ confidence (dashed line in Fig. 3). In X-rays and VHE $\gamma$ rays, the sampling is sparse and prevents measurement of any trend. The trend seen in radio and optical suggests a common large, emission region for these wavebands. As it is known that most of the emission at $15 \mathrm{GHz}$ originates in the parsec scale

\footnotetext{
8 For details on the background models, see http://fermi.gsfc. nasa.gov/ssc/data/access/lat/BackgroundModels.html

9 The test statistic (Mattox et al. 1996) is defined as TS= 2( $\left.\log L_{1}-\log L_{0}\right)$, where $L$ is the likelihood of the data given the model with $\left(L_{1}\right)$ or without $\left(L_{0}\right)$ a point source in the chosen position.
} 


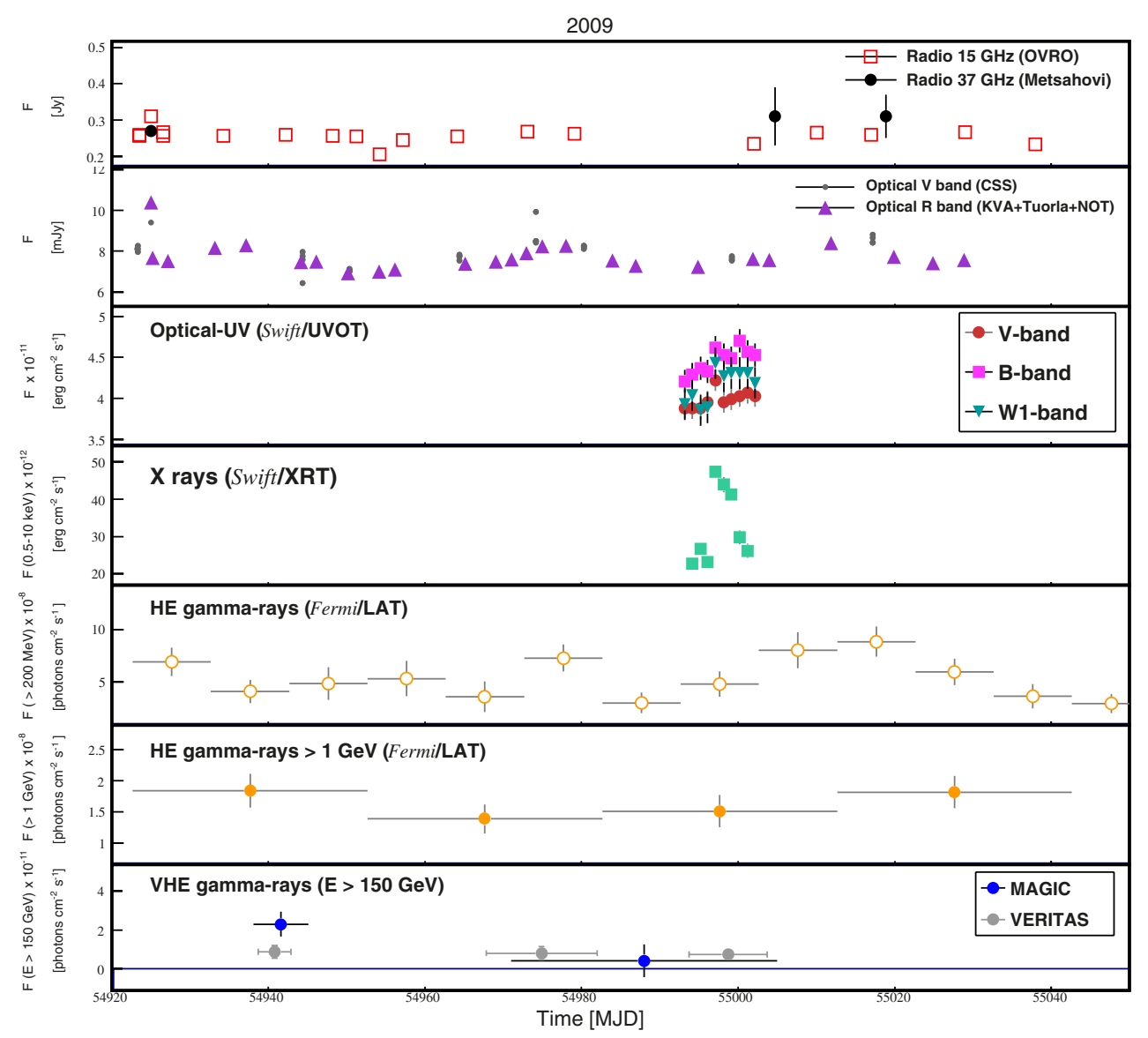

Fig. 4. Zoom of multiwavelength light curve of PKS 1424+240 around the X-ray flare in 2009.

jet (see Sect. 3.1), this also plays a significant part of the optical emission there. However, in addition to the long-term trend, it is evident that the optical light curve also shows faster flares.

Figure 4 shows the light curves in 2009. There is fast X-ray flare observed in June 2009 and around this time, the flux in UVOT band also starts to increase. However, the increase is much slower and also smaller in amplitude, and therefore, it is not clear if it has common origin with the X-ray flare.

In the radio bands, the time coverage of the measurements does not allow any conclusion, and in VHE and HE $\gamma$ rays, the simultaneous time bins do not show short-term variability. Interestingly, there also seems to be a simultaneous increase of optical and HE $\gamma$-ray flux above $200 \mathrm{MeV}$ at around the time when there was an X-ray outburst (MJD 54995), which might suggest a common origin for the optical and HE $\gamma$-ray emission. However, this trend is not significant in HE $\gamma$ rays when considering the entire data sample.

In addition to visual inspection of the light curves, we also perform a simple correlation study between different bands. The only two bands showing a possible correlation are the optical and radio $(15 \mathrm{GHz})$ wavelengths: in Fig. 5, the data pairs of the $R$-band and $15 \mathrm{GHz}$ with a time difference $<0.9$ days are plotted against each other. Although the linear fit has a low probability, it has a Spearman correlation coefficient of $81 \%$ and, in a likelihood ratio test, it is significantly (>99.9\%) preferred over constant fit. The correlation is dominated by the long-term trend. We did not see any significant correlation when considering timescales shorter than one year. This result suggests that the radio and optical emission have a component in common that originates in a source, which varies on $\sim 1$-year

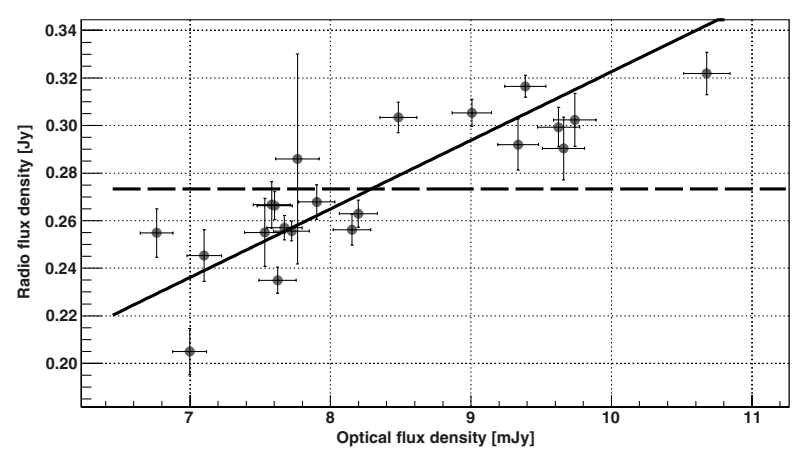

Fig. 5. Optical $R$-band flux versus radio $15 \mathrm{GHz}$ flux. The data pairs are selected with a time difference of $<0.9$ days. The linear fit is significantly preferred over the constant fit (see text for details).

timescales. A confirmation of this statement will not be possible until radio/optical data are accumulated over many more years.

\subsection{Spectral energy distribution}

Using the multiwavelength data discussed in the previous subsections, the SED of PKS 1424+240 was constructed from VHE $\gamma$ rays to the radio band. For VHE $\gamma$ rays, we plot the data from the 2009, 2010, and 2011 observations, which is corrected for the EBL absorption using the Franceschini et al. (2008) model. The EBL model of Domínguez et al. (2011) gives compatible results. The spectrum from Fermi-LAT covers the whole 2008-2011 period. In X-rays, we use two spectra from Swift from 2009, one from a high state (MJD 54997) and one 


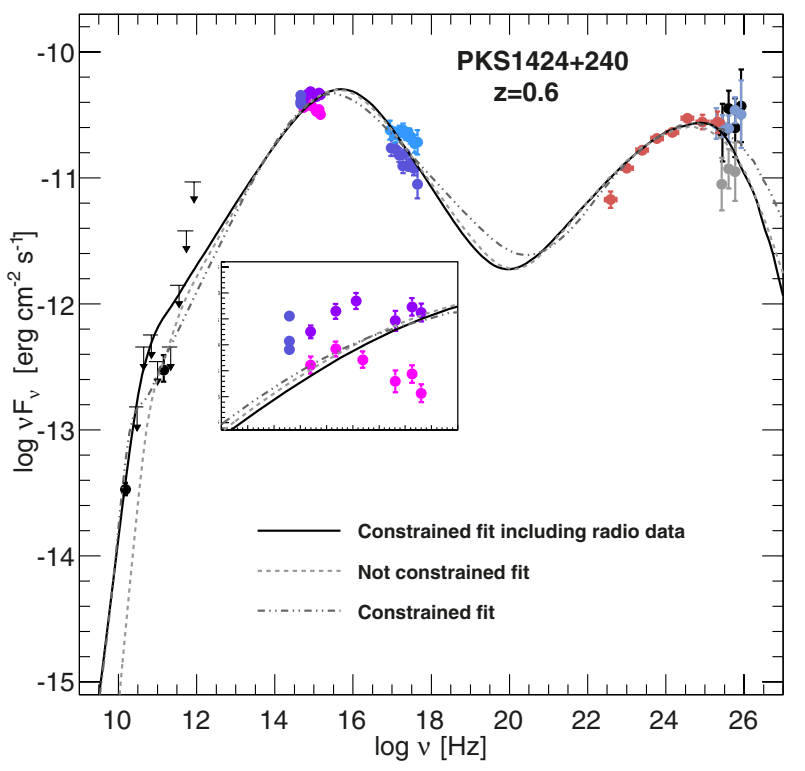

Fig. 6. SED of PKS $1424+240$ constructed from radio to VHE $\gamma$ rays: radio $15 \mathrm{GHz}$ from OVRO (black filled circles), $30 \mathrm{GHz}$ to $857 \mathrm{GHz}$ from Planck (black arrows and filled circle), optical $R$-band from KVA (blue filled circles), optical to UV from UVOT (pink (lowest state) and purple (highest state) filled circles), X-rays from Swift-XRT (light blue (high state) and violet (low state) filled circles), HE $\gamma$ rays from FermiLAT (red filled circles) and VHE $\gamma$ rays from MAGIC (2009: black, 2010: gray, 2011: light blue). The SED is fitted with three single-zone SSC models: the high $\gamma_{\min }$ fit (solid line) and the fits that result from the $\chi^{2}$ minimization (dashed and dot-dashed lines; see text for details). The inset shows the optical-UV range of the SED on an expanded scale. A redshift of $z=0.6$ is assumed.

from a low state (MJD 54 996), and simultaneous UVOT data for these epochs. The $R$-band points correspond to the average flux in 2009, 2010, and 2011 during the MAGIC observations. In the radio band, we subtract the contribution from the extended jet (see Sect. 3.1.) from the OVRO $15 \mathrm{GHz}$ measurement and plot the average flux between 2009 and 2011. Additionally, we use the Planck data from January-February 2010 that are published in Giommi et al. (2012). The data are not simultaneous, but together they present a low and high state in different wavebands.

The SED of PKS 1424+240 shows a wide synchrotron bump peaking around the optical regime ${ }^{10}$. The location of the second peak is more uncertain but seems to be located at very high energies, making the separation between the first and second peak large. This feature is difficult to model with a traditional single zone SSC. Additionally, the multiwavelength variability suggests a possible correlation between radio and optical fluxes, which means that the emission could originate in the VLBA core for which the Doppler factor and the size of the emitting region were derived from the MOJAVE data (see Sect. 3.1). Therefore, in addition to a traditional one-zone model (Tavecchio et al. 1998; Maraschi et al. 2003; Fig. 6), we also explore the twozone model of (Tavecchio et al. 2011; Fig. 7). The parameters of the fits are presented in Table 3.

In the canonical one-zone SSC model, the emitting plasma is contained in a spherical region of radius $R$ in relativistic motion (described by a bulk Lorentz factor $\Gamma$ ) along the jet at an angle $\theta$ with respect to the line of sight to the observer, so that

\footnotetext{
${ }^{10}$ In the SED shown in Fig. 4 of Acciari et al. (2010), the synchrotron peak seems to be narrower due to lower (possibly erroneously reproduced) X-ray luminosity.
}

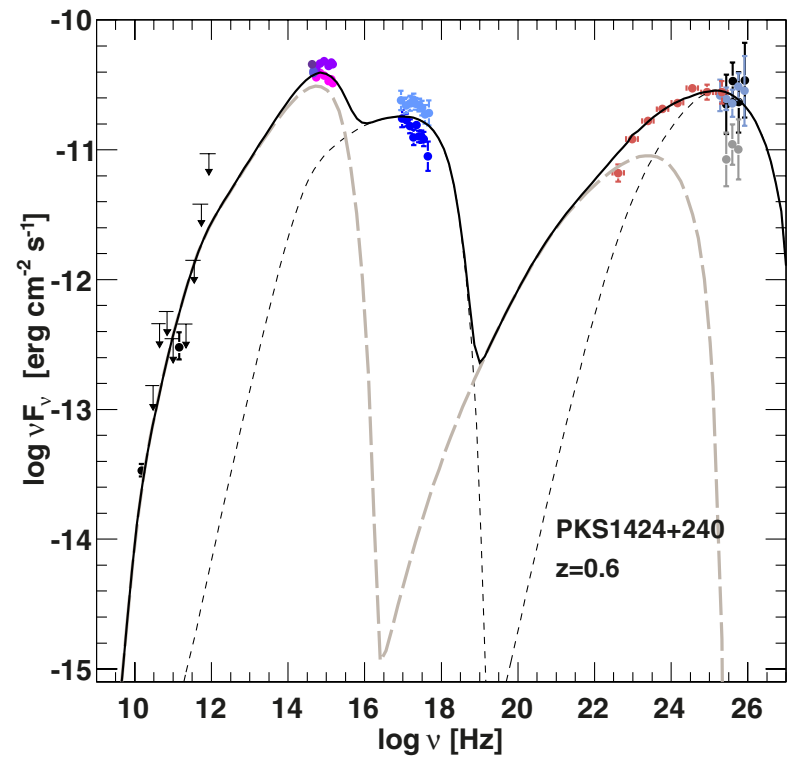

Fig. 7. SED of PKS $1424+240$ fitted with a two-zone SSC model assuming a redshift of $z=0.6$. The long dashed line represents the emission from the outer region and the dashed line from the inner region (see text). Data as in Fig. 6.

special relativistic effects are cumulatively described by the relativistic Doppler factor, $\delta=[\Gamma(1-\beta \cos \theta)]^{-1}$. The emitting region is filled with a homogeneous tangled magnetic field with intensity $B$ and by a population of relativistic electrons of density $n_{\mathrm{e}}$, whose spectrum is described by a broken power law as a function of the energy of the relativistic electrons:

$N(\gamma)=K \gamma^{-n_{1}}\left(1+\frac{\gamma}{\gamma_{\mathrm{b}}}\right)^{n_{1}-n_{2}}$,

where $K$ is the normalization factor, $\gamma_{\mathrm{b}}$ is the Lorentz factor of electrons at the spectral break and $n_{1}$ and $n_{2}$ the spectral indices below and above the break, respectively.

As a first approach, we fit the SED excluding the radio data (assuming that the radio emission originates in a different region that does not contribute to the emission in other energy regimes). The fit is performed using the fully automatized $\chi^{2}$-minimization procedure of Mankuzhiyil et al. (2011). The systematic errors on the flux were estimated to be $2 \%, 10 \%$, and $40 \%$ for radiooptical-X-ray, GeV $\gamma$ rays, and VHE $\gamma$ rays, respectively. The resulting fit is shown with a dashed line in Fig. 6. While reproducing the high-energy SED data reasonably well, it fails in reproducing the shape of the optical-UV continuum (inset of Fig. 6). If the radio-optical connection can be confirmed with long-term study, this model is disfavored because the radio emission is assumed to be produced in a different part of the jet.

As a second approach, we include radio data in the modeling by considering the possible connection we found between radio and optical wavebands.

However, it is not possible to reproduce the SED using a Doppler factor of $\sim 10$, such as that derived from the VLBA data, by assuming typical viewing angles of $1-5^{\circ}$ (see Sect. 3.1). The Doppler factor found from fitting the SED is an order of magnitude higher than the VLBA-derived value (dashed-dotted line fit of Fig. 6). Even when constraining the Doppler factor to 40 in the fitting procedure, the low energy peak data cannot be properly described by the model (continuous line of Fig. 6). Moreover, this last model cannot fulfill all the requirements. In particular, the variability timescale of one day observed during 
Table 3. Model parameters for the four models.

\begin{tabular}{|c|c|c|c|c|c|c|c|c|c|c|c|c|}
\hline Model & $\begin{array}{c}\gamma_{\min } \\
{\left[10^{3}\right]}\end{array}$ & $\begin{array}{c}\gamma_{\mathrm{b}} \\
{\left[10^{4}\right]}\end{array}$ & $\begin{array}{l}\gamma_{\max } \\
{\left[10^{5}\right]}\end{array}$ & $n_{1}$ & $n_{2}$ & $\begin{array}{c}B \\
{[\mathrm{G}]}\end{array}$ & $\begin{array}{c}K \\
{\left[\mathrm{~cm}^{-3}\right]}\end{array}$ & $\begin{array}{c}R \\
{\left[10^{16} \mathrm{~cm}\right]}\end{array}$ & $\delta$ & $\begin{array}{c}L_{\mathrm{kin}(\mathrm{p})} \\
{\left[10^{45} \mathrm{erg} \mathrm{s}^{-1}\right]}\end{array}$ & $\begin{array}{c}L_{\mathrm{kin}(e)} \\
{\left[10^{45} \mathrm{erg} \mathrm{s}^{-1}\right]}\end{array}$ & $\begin{array}{c}L_{B} \\
{\left[10^{43} \mathrm{erg} \mathrm{s}^{-1}\right]}\end{array}$ \\
\hline $\begin{array}{l}\text { One-zone } \\
\text { (No radio) }\end{array}$ & 0.260 & 3.2 & $8.9 \times 10^{3}$ & 1.9 & 3.9 & 0.018 & $2 \times 10^{2}$ & 6.5 & 70 & 5 & 7.0 & 3 \\
\hline One-zone & 0.016 & 2.6 & $3.9 \times 10^{2}$ & 1.7 & 3.7 & 0.006 & 50 & 5 & 131 & 64 & 21 & 0.8 \\
\hline $\begin{array}{l}\text { One-zone } \\
\text { (Constrained) }\end{array}$ & 0.004 & 5.3 & $3.2 \times 10^{4}$ & 2.0 & 4.0 & 0.017 & $1.7 \times 10^{2}$ & 19 & 40 & 371 & 11 & 8.8 \\
\hline 2 zones (in) & 8.0 & 3.9 & 7.0 & 2.0 & 3.1 & 0.033 & $3.1 \times 10^{3}$ & 4.8 & 30 & 0.07 & 1.2 & 1.1 \\
\hline 2 zones (out) & 0.6 & 3.0 & 0.5 & 2.0 & 3.0 & 0.033 & 23 & 190 & 9 & 1.3 & 2.3 & 159 \\
\hline
\end{tabular}

Notes. The four models are: one-zone model without considering radio data, one-zone model including the radio data, one-zone model including the radio data and considering constraints derived from the VLBA data, and two-zone model where the outer emission region considers the constraints derived from the VLBA data. The following quantities are reported: the minimum, break, and maximum Lorentz factors $\left(\gamma_{\min }, \gamma_{\mathrm{b}}\right.$, $\left.\gamma_{\max }\right)$; the low and high-energy slope of the electron energy distribution $\left(n_{1}\right.$ and $\left.n_{2}\right)$; the magnetic field intensity; $B$, the electron density; $K$, the radius of the emitting region, $R$; the Doppler factor, $\delta$; and the kinetic energy of the protons, electrons, and magnetic field $\left(L_{\mathrm{kin}(\mathrm{p})}, L_{\mathrm{kin}(\mathrm{e})}\right.$, and $\left.L_{B}\right)$.

the X-ray flare in 2009 cannot be attained. The causality relation $\left(R<c t_{\mathrm{var}} \delta /(1+z)\right)$ gives $R<6.5 \times 10^{16} \mathrm{~cm}$ in this case, while the fit parameter requires $R \sim 19 \times 10^{16} \mathrm{~cm}$. It, therefore, seems that the emission zone parameters do not agree with the ones derived for the VLBA core in the one-zone modeling. This is problematic because all the emission in a one zone model should originate in the same region, which, basing on the radio emission, could be placed in the VLBA core.

For the two-zone modeling, we adopt the model presented for the flat-spectrum radio quasar PKS $1222+216$ in Tavecchio et al. (2011) with small modifications. We neglect the external seed photon contributions as PKS1424+240 is a BL Lac object where the disk and broad-line region are assumed to be weak, and therefore, external Compton models are not appealing. The two emission regions are separated: the inner region is assumed to be smaller. This could mean that it is closer to the central black hole or that it is a spine, while the larger region is a sheath. For the larger emission region, the radius $R$ is equal to half the cross-sectional size of a conical jet. Both regions are described by the same parameters as the one-zone model, assuming an electron population distributed as a broken power-law in each region. Since the cooling time of the electrons at the highest energies is shorter than the light-crossing time (i.e. the escape from the source), the absence of a break in the electron distribution requires a continuous acceleration of high-energy electrons. The SED is modeled with two emission regions such that the larger emission region would have the properties observed for the VLBA core. The resulting fit is shown in Fig. 7, and the adopted parameters are in the last line of Table 3. The two-zone model results in an acceptable fit to the data. The high-energy part of the SED model Fig. 8 reveals that in this case the peak of the second bump is located at $\sim 60 \mathrm{GeV}$. The high $\gamma_{\text {min }}$ obtained is compatible with the hypothesis of shock heating, as suggested, for example, in Virtanen \& Vainio (2003) and Sironi \& Spitkovsky (2011).

The measured powers indicate a strange behavior of the magnetic field, whose relative importance compared to kinetic energy of particles increases with the distance. For a very different radius $R$, we obtain the same magnetic field $B$, meaning a very large magnetic energy in the large zone. The behavior of the bulk Lorentz factor $\Gamma$ suggests some deceleration of the flow (e.g. Georganopoulos \& Kazanas 2003) which could explain the magnetic field increase (through compression). This behavior, however, is not sufficient to explain the values of the model. Assuming a steady jet under expansion, $B$ is

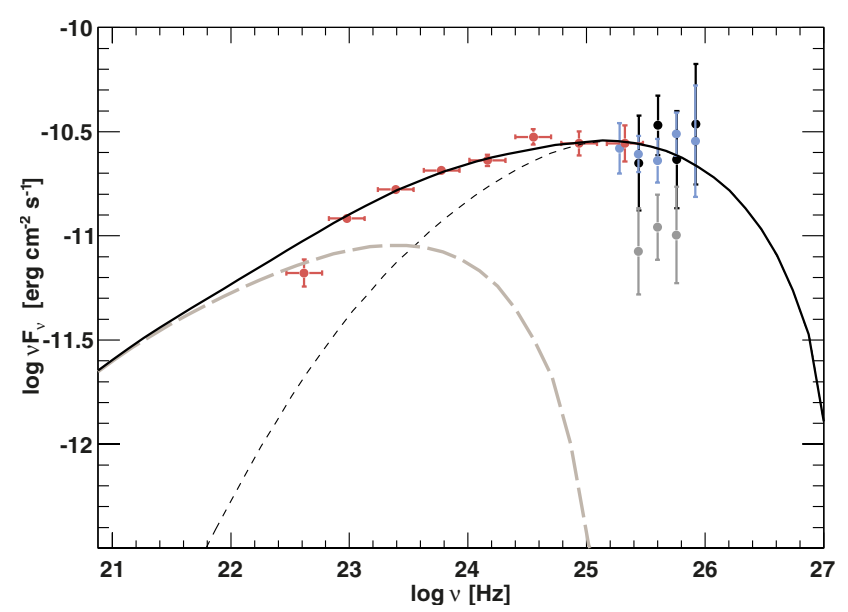

Fig. 8. High-energy part of the SED of PKS $1424+240$ fitted with a twozone SSC model and with Fermi-LAT data (red symbols) and MAGIC 2009, 2010, and 2011 data (gray, black, and blue symbols, respectively), which are corrected for EBL absorption by assuming redshift of 0.6 and the EBL model of Franceschini et al. (2008). The peak is located at $\sim 60 \mathrm{GeV}$.

expected to scale as $R^{-1}$. Then the relation between the magnetic field and the radius of the inner and outer regions should be $B_{\text {out }} / B_{\text {in }}=R_{\text {in }} / R_{\text {out }}=0.025$. Deceleration could in principle compensate for this decrease, since $B \propto \Gamma^{-1}$ through compression if the decelerating jet propagates cylindrically. In the case of PKS $1424+240$, if we consider that the viewing angle $\theta_{\text {in }}=1 / \Gamma_{\text {in }}$, then $\Gamma_{\text {in }}=\gamma_{\text {in }}$, while the viewing angle of the outer region would be $\theta_{\text {out }}=1.91^{\circ}$ and therefore, $\Gamma_{\text {out }}=$ 4.66. The relation among the two emitting regions would be $B_{\text {out }} / B_{\text {in }}=\Gamma_{\text {in }} / \Gamma_{\text {out }}=6.44$, which is too small (by a factor of 10) to compensate for the expansion. A possible explanation is a jet with primary jet injection that changes parameters with time. Alternatively, if the two regions are not located at different distances along the jet but are cospatial and form a spine-layer structure (e.g. Ghisellini et al. 2005), it is then possible that the magnetic field is uniform in the jet, which explains the same value in the fast and slow region.

\section{Summary and discussion}

In this paper, we present the first long-term study of the VHE $\gamma$-ray emission from the BL Lac object PKS 1424+240. 
The redshift of this source is uncertain. Recently, a lower limit of the redshift $z \geq 0.6035$ was determined from the $\operatorname{Ly} \beta$ and $\operatorname{Ly} \gamma$ absorption of the intergalactic medium by Furniss et al. (2013), making the source a strong candidate to be the farthest known VHE $\gamma$-ray emitter.

MAGIC observed the source for three years from 2009 to 2011. We did not find any significant $\gamma$-ray excess above $400 \mathrm{GeV}$, while VERITAS did (see Acciari et al. 2010), but this could be due to the shorter MAGIC observations (in comparison to those from VERITAS). The upper limit on the flux at $500 \mathrm{GeV}$ reported in Fig. 1 agrees with the value reported by Acciari et al. (2010) at the same energy. The $2 \sigma$ confidence upper limit for the redshift, $z<0.81$, derived from the MAGIC 2011 spectrum (which is the most precise among the MAGIC spectra) and the Franceschini et al. (2008) EBL model, agrees with the new lower limit.

Furniss et al. (2013) reported that the VERITAS VHE spectrum (corrected for EBL absorption) exhibited a lower flux than an extrapolation from the Fermi-LAT power-law spectrum in all but the highest energy bin, which was interpreted as an overestimation of the $\gamma$-ray opacity or the onset of an unexpected VHE spectral feature. In this work, we used the average 2008-2011 Fermi-LAT spectrum, which extends to higher energies and has smaller statistical error bars than the 2009 Fermi-LAT spectrum that is simultaneous to the VERITAS observations used in Furniss et al. (2013). The 3-year Fermi-LAT spectrum extends beyond $100 \mathrm{GeV}$ and connects smoothly (within uncertainties) with the 2009, 2010, and 2011 MAGIC spectra.

The VHE light curve of the source is compatible with the hypothesis of constant flux at a 95\% confidence level, and the spectral index of the differential energy spectrum is steady within the error bars. The MAGIC VHE $\gamma$-ray observations in 2009 and 2011 were triggered by the optical outbursts in the source, but we find no conclusive evidence for a higher VHE $\gamma$-ray flux from the source during the optical outbursts in yearly timescales. The two periods that were triggered by optical outburst showed moderately higher VHE $\gamma$-ray flux than the 2010 period. However, the average optical flux was higher in 2011, while the highest VHE $\gamma$-ray flux was measured in 2009. As the source is rather weak in the VHE $\gamma$-ray band and the observations of several nights have to be combined in the light curve, a detailed short term comparison is not possible.

We also present the first long-term multiwavelength dataset of the source that covers 2006-2011 and includes data from radio to HE $\gamma$ rays. The source is variable in all studied energy regimes, although the variability is moderate. We find a significant increasing trend in the $15 \mathrm{GHz}$ radio and optical light curves in 2009-2011, as well as correlation between the two bands, which we interpret as a common large, emission region, which possibly could be the parsec scale jet. We investigate the MOJAVE $15 \mathrm{GHz}$ VLBA observations of the source and find that the parsec scale jet is slow and therefore could be responsible for such long-term variability. The common origin of radio and optical emission in blazars has been studied extensively in the past (e.g. Tornikoski et al. 1994; Hanski et al. 2002). The results have been inconclusive with some sources showing correlations while others not. Here, we find a clear indication of connection between these two energy regions in a high synchrotron peaking source. Sources of this type are weak in the radio band, and therefore, such a connection has not been considered in the past studies. We emphasize the need for further investigation of this connection in a sample of sources.

We also investigate the SED of the source with a nonsimultaneous dataset that presents the low and high state of the source within 2009-2011. The one-zone fit to the SED (both including and excluding the radio data) fails to reproduce the optical-UV continuum and requires a much higher Doppler factor than that observed in the parsec scale jet by the VLBA. Even when constraining the Doppler factor in the fitting procedure, no result is derived for a Doppler factor lower than 40. The same mismatch in Doppler factors has been seen for many VHE $\gamma$-ray emitting BL Lac objects (see, e.g., Tiet et al. 2012, and references therein). In many of the sources, the high Doppler factor of the VHE $\gamma$-ray emitting region is required by the fast variability seen in the VHE $\gamma$-ray regime (Albert et al. 2007; Aharonian et al. 2007). This indicates separate emission regions for radio and $\gamma$-ray emission, and it has been suggested that jets are deccelerating (Georganopoulos \& Kazanas 2003) or have spine-sheath structure (Ghisellini et al. 2005). In the case of PKS 1424+240, the one-day scale variability is only observed in X-rays, which limits the size of the emission region to $\sim 1.6 \times 10^{15} \delta[\mathrm{cm}]$. Given the multiple pieces of evidence in favor of two emission zones, we model the SED with the twozone model. According to this model, the radio and majority of the optical emission originate in a large emission region, and the $\mathrm{X}$-ray to $\gamma$-ray emission originates mainly in a smaller emission region with a minor contribution from the larger region in the Fermi-LAT energy band. The two-zone model has twice as many parameters and fits the SED better than the single zone models. However, this model requires a high $\gamma_{\min }$ for the inner region that produces the $\gamma$-ray emission to not overproduce the synchrotron emission in the optical and radio bands. As discussed in Aleksić et al. (2012b), high $\gamma_{\min }$ values have also been adopted for other blazars, like 1ES $1215+303$ and the so-called extreme BL Lacs (that show very hard X-ray spectra; Katarzynski et al. 2005; Tavecchio et al. 2009; Kaufmann et al. 2011; Lefa et al. 2011), and are consistent with simulation results (Virtanen \& Vainio 2003; Sironi \& Spitkovsky 2011). Other evidence in favor of the two-zone model is that the $\mathrm{X}$-ray flare is less visible in $V$ and $R$-bands. The model can account for this feature, since a higher flux in the inner (small) component will mainly affect UV frequencies and higher frequencies in the optical, while the $V$ and $R$-bands are more dominated by the emission from the larger component.

Based on the long-term multiwavelength light curve studies and the SED modeling, we conclude that the two emission region model is favored for PKS 1424+240, while the previous work (Acciari et al. 2010) concluded that the one-zone SSC model is sufficient for describing the SED. This shows that inclusion of long-term multiwavelength light curves from radio to $\gamma$ rays gives significant input for the modeling of the emission. However, the SED studies here were done with the nonsimultaneous data, and we emphasize the need for strictly simultaneous multiwavelength observations for this source as a starting point for further studies.

Acknowledgements. We are grateful to the anonymous referee for the valuable comments and suggestions. We would like to thank the Instituto de Astrofisica de Canarias for the excellent working conditions at the Observatorio del Roque de los Muchachos in La Palma. The support of the German BMBF and MPG, the Italian INFN and Spanish MICINN is gratefully acknowledged. This work was also supported by ETH Research Grant TH 34/043, by the Polish MNiSzW Grant N N203 390834, by the YIP of the Helmholtz Gemeinschaft, and by grant DO02-353 of the the Bulgarian National Science Fund. Partly based on observations made with the Nordic Optical Telescope, operated on the island of La Palma jointly by Denmark, Finland, Iceland, Norway, and Sweden, in the Spanish Observatorio del Roque de los Muchachos of the Instituto de Astrofisica de Canarias. The data presented here were obtained in part with ALFOSC, which is provided by the Instituto de Astrofisica de Andalucia (IAA) under a joint agreement with the University of Copenhagen and NOTSA. The CSS survey is funded by the National Aeronautics and Space Administration under 
Grant No. NNG05GF22G issued through the Science Mission Directorate NearEarth Objects Observations Program. The CRTS survey is supported by the US National Science Foundation under grants AST-0909182. The OVRO 40-m monitoring program is supported in part by NASA grants NNX08AW31G and NNX11A043G, and NSF grants AST-0808050 and AST-1109911. The Fermi LAT Collaboration acknowledges generous ongoing support from a number of agencies and institutes that have supported both the development and the operation of the LAT as well as scientific data analysis. These include the National Aeronautics and Space Administration and the Department of Energy in the United States, the Commissariat à l'Énergie Atomique and the Centre National de la Recherche Scientifique/Institut National de Physique Nucléaire et de Physique des Particules in France, the Agenzia Spaziale Italiana and the Istituto Nazionale di Fisica Nucleare in Italy, the Ministry of Education, Culture, Sports, Science and Technology (MEXT), High Energy Accelerator Research Organization (KEK) and Japan Aerospace Exploration Agency (JAXA) in Japan, and the K. A. Wallenberg Foundation, the Swedish Research Council and the Swedish National Space Board in Sweden. Additional support for science analysis during the operations phase is gratefully acknowledged from the Istituto Nazionale di Astrofisica in Italy and the Centre National d'Études Spatiales in France.

\section{References}

Abdo, A. A., Ackermann, M., Ajello, M., et al. 2009, ApJ, 707, 1310 Abdo, A. A., Ackermann, M., Ajello, M., et al. 2010a, ApJS, 188, 405 Abdo, A. A., Ackermann, M., Agudo, I., et al. 2010b, ApJ, 716, 30 Acciari, V. A., Aliu, E., Arlen, T., et al. 2010, ApJ, 708, L100 Ackermann, M., Ajello, M., Albert, A., et al. 2012, ApJS, 203, 4 Aharonian, F. 2000, New Astron., 5, 377

Aharonian, F., Akhperjanian, A. G., Bazer-Bachi, A. R., et al. 2007, ApJ, 664, 71

Albert, J., Aliu, E., Anderhub, H., et al. 2007, ApJ, 669, 862

Albert, J., Aliu, E., Anderhub, H., et al. 2008a, ApJ, 674, 1037

Albert, J., Aliu, E., Anderhub, H., et al. 2008b, Nucl. Instrum. Meth. Phys. Res. A, 594, 407

Albert, J., Aliu, E., Anderhub, H., et al. 2008c, Nucl. Instrum. Meth. Phys. Res. A, 588, 424

Albert, J., Aliu, E., Anderhub, H., et al. 2008d, ApJ, 674, 1037

Aleksić, J., Antonelli, L. A., Antoranz, P., et al. 2010, A\&A, 524, A77

Aleksić, J., Antonelli, L. A., Antoranz, P., et al. 2011, ApJ, 729, 115

Aleksić, J., Alvarez, E. A., Antonelli, L. A., et al. 2012a, Astropart. Phys., 35, 435

Aleksić, J., Alvarez, E. A., Antonelli, L. A., et al. 2012b, A\&A, 544, A142 Aliu, E., Anderhub, H., Antonelli, L. A., et al. 2008, Science, 322, 1221 Aliu, E., Anderhub, H., Antonelli, L. A., et al. 2009, Astropart. Phys., 30, 293 Aller, M. F., Aller, H. D., \& Hughes, P. A. 1992, ApJ, 339, 1

Archambault, S., Aune, T., Behera, B., et al. 2014, ApJ, 785, L16

Atwood, W. B., Abdo, A. A., Ackermann, M., et al. 2009, ApJ, 697, 1071

Baixeras, C., Bastieri, D., Bigongiari, C., et al. 2004, Nucl. Instrum. Phys. Res.

A, 518, 188

Baars, J. W. M., Baars, J. W. M., Genzel, R., et al. 1977, A\&A, 61, 99

Barthelmy, S. D., Barbier, L. M., Cummings, J. R., et al. 2005, Space Sci. Rev., 120,143

Burrows, D. N., Hill, J. E., Nousek, J. A., et al. 2005, Space Sci. Rev., 120, 165 Conrad, J. http://root. cern. ch/root/html510/TRolke.html, 2004

Cortina, J., Goebel, F., \& Schweizer, T. 2009, Contribution to the 31st ICRC, Lodz, Poland, July 2009, submitted [arXiv: 0907. 1211]

Cusumano, G., La Parola, V., Segreto, A., et al. 2010, A\&A, 524, A64

Danforth, C. W., Keeney, B. A., Stocke, J. T., et al. 2010, ApJ, 720, 976

Domínguez, A., Primack, J. R., Rosario, D. J., et al. 2011, MNRAS, 410, 2556

Drake, A. J., Djorgovski, S. G., Mahabal, A., et al. 2009, ApJ, 696, 870

Fanti, R., Fanti, C., Fanti, R., et al. 1974 A\&ASS, 18, 147

Finke, J. D., Razzaque, \& S. Dermer, C. D. 2010, ApJ, 712, 1, 238

Fiorucci, M., \& Tosti, G. 1996, A\&AS, 116, 403

Fomin, V. P., Stepanian, A. A., Lamb, R. C., et al. 1994, Astropart. Phys. 2, 137

Franceschini, A., Rodighiero, G., \& Vaccari, M. 2008, A\&A, 487, 837

Furniss, A., Williams, D. A., Danforth, C., et al. 2013, ApJ, 768, 31

Georganopoulos, M., \& Kazanas, D. 2003, ApJ, 594, 27

Ghisellini, G., Tavecchio, F., \& Chiaberge, M. 2005, A\&A, 432, 401

Gilmore, R. C., Gilmore, R. C., Madau, P., et al. 2009, MNRAS, 399, 1694

Giommi, P., Polenta, G., Lähteenmäki, A., et al. 2012, A\&A, 541, A160

Hauser, M. G., \& Dwek, E. 2001, ARA\&A, 39, 249

Hanski, M., Takalo, L. O., \& Valtaoja, E. 2002, A\&A, 394, 17

Hillas, A. M. 1985, in International Cosmic Ray Conference, ed. F. C. Jones, 3, 445

Hovatta, T., Valtaoja, E., Tornikoski, M., et al. 2009, A\&A, 494, 527

Impey, C. D., \& Tapia, S. 1988, ApJ, 333, 666

Impey, C. D., \& Tapia, S. 1990, ApJ, 354, 124
Isobe, T., Feigelson, E. D., Akritas, M. G., et al. 1990, ApJ, 364, 104 Jannuzi, B. T., Green, R. F., \& French, H. 1993, ApJ, 404, 100

Kalberla, P. M. W., Burton, W. B., Hartmann, D., et al. 2005, A\&A, 440, 775

Katarzynski, K., Sol, H., \& Kus, A. 2001, A\&A, 367, 809

Katarzynski, K., Ghisellini, G., Tavecchio, F., et al. 2005, A\&A, 433, 479

Kaufmann, S., Wagner, S. J., Tibolla, O., et al. 2011, A\&A, 534, A130

Kneiske, T. M., \& Dole, H. 2010, A\&A, 515, A19

Lefa, E., Rieger, F. M., \& Aharonian, F. 2011, ApJ, 740, 64

Li, T. P., \& Ma, Y. Q. 1983, ApJ, 272, 317

Lister, M., Aller, M. F., Aller, H., et al. 2011, ApJ, 742, 27

Mankuzhiyil, N., Ansoldi, S., Persic, M., et al. 2011, ApJ, 733, 14

Mannheim, K., \& Biermann, P. L. 1992, A\&A, 253, L21

Mattox, J. R., Bertsch, D. L., Chiang, J., et al. 1996, ApJ, 461, 396

Maraschi, L., \& Tavecchio, F. 2003, ApJ, 593, 667

Maraschi, L., Ghisellini, G., \& Celotti, A. 1992, ApJ, 397, L5

Mead, A. R. G., Ballard, K. R., Brand, P. W. J. L., et al. 1990, A\&AS, 83, 183

Meisner, A. M., \& Romani, R. W. 2010, ApJ, 712, 14

Nolan, P., Abdo, A. A., \& Ackermann, M., et al. 2012, ApJS, 199, 31

Ong, R. (the VERITAS Collaboration) 2009, ATel\#2084

Pei, Y. C. 1992, ApJ, 395, 130

Piner, B. G., Pant, N., Edwards, Ph. G., et al. 2009, ApJ, 690, 31

Pita, S., Goldoni, P., Boisson, C., et al. 2012, AIP Conf. Proc., 1505, 566

Poole, T. S., Breeveld, A. A., Page, M. J., et al. 2008, MNRAS, 383, 627

Prandini, E., Bonnoli, G., Maraschi, L., et al. 2010, MNRAS, 405, L76

Prandini, E., Mariotti, F., Tavecchio, F., et al. 2011, Fermi Symp. Proc., eConf C110509 [arXiv: 1111.0913]

Pushkarev, A. B., Kovalev, Y. Y., Lister, M. L., et al. 2009, A\&A, 507, L33

Rau, A., Schady, P., Greiner, J., et al. 2012, A\&A, 538, A26

Reimer, A. 2012, Proc. International Workshop on Beamed and Unbeamed Gamma-Rays from Galaxies, J. Phys. Conf. Ser., 355, 2011

Richards, et al. 2014, MNRAS, in press

Richards, J. L., Max-Moerbeck, W., Pavlidou, V., et al. 2011, ApJS, 194, 29

Rolke, W. A., Lopez, A. M., \& Conrad, J. 2005, NIM A551, 493

Roming, P. W. A., Kennedy, T. E., Mason, K. O., et al. 2005, Space Sci. Rev., 120,95

Savolainen, T., Homan, D. C., Hovatta, T., et al. 2010, A\&A, 512, A24

Sbarufatti, B., Treves, A., \& Falomo, R. 2005, ApJ, 635, 173

Scarpa, R., \& Falomo, R. 1995, A\&A, 303, 656

Schlegel, D. J., Finkbeiner, D. P., \& Davis, M. 1998, ApJ, 500, 525

Shaw, M., Romani, R. W., Healey, S. E., et al. 2009, ApJ, 704, 477

Shaw, M., Romani, R. W., Cotter, G., et al. 2013, ApJ, 764, 135

Sironi, L., \& Spitkovsky, A. 2011, ApJ, 726, 75

Stecker, F. W., Malkan, M. A., \& Scully, S. T. 2006, ApJ, 648, 774

Stecker, F. W., Malkan, M. A., \& Scully, S. T. 2012, ApJ, 761, 128

Tavecchio, F., Maraschi, L., \& Ghisellini, G. 1998, ApJ, 509, 608

Tavecchio, F., Ghisellini, G., Ghirlanda, G., et al. 2009, MNRAS, 399, 59

Tavecchio, F., Ghisellini, G., Ghirlanda, G., et al. 2010, MNRAS, 401, 1570

Tavecchio, F., Becerra-Gonzalez, J., Ghisellini, G., et al. 2011, A\&A, 534, A86

Teräsranta, H., Tornikoski, M., Mujunen, A., et al. 1998, A\&AS, 132, 305

Teshima, M. (the MAGIC Collaboration), ATel\#2098

Tiet, V. C., Piner, B. G., \& Edwards, P. G. 2012, in Proc. Fermi \& Jansky 2012 eConf C1111101 [arXiv: 1205.2399]

Tornikoski, M., Valtaoja, E., Terasranta, H., et al. 1994, A\&A, 289, 673

Treves, A., Falomo, R., \& Uslenghi, M. 2007, A\&A, 473, L17

Urry, C. M., \& Padovani, P. 1995, PASP, 107, 803

Virtanen, J., \& Vainio, R. 2003, Proc. High Energy Blazar Astronomy, eds. L. O. Takalo, \& E. Valtaoja, ASP Conf. Proc., 299, 157

1 IFAE, Campus UAB, 08193 Bellaterra, Spain

2 Università di Udine, and INFN Trieste, 33100 Udine, Italy

3 INAF National Institute for Astrophysics, 00136 Rome, Italy

4 Università di Siena, and INFN Pisa, 53100 Siena, Italy

5 Croatian MAGIC Consortium, Rudjer Boskovic Institute, University of Rijeka and University of Split, 10000 Zagreb, Croatia

6 Max-Planck-Institut für Physik, 80805 München, Germany

7 Universidad Complutense, 28040 Madrid, Spain

8 Inst. de Astrofísica de Canarias, 38200 La Laguna, Tenerife, Spain

9 University of Łódź, 90236 Lodz, Poland

10 Deutsches Elektronen-Synchrotron (DESY), 15738 Zeuthen, Germany

11 ETH Zurich, 8093 Zurich, Switzerland

12 Universität Würzburg, 97074 Würzburg, Germany

13 Centro de Investigaciones Energéticas, Medioambientales y Tecnológicas, 28040 Madrid, Spain

14 Technische Universität Dortmund, 44221 Dortmund, Germany 
15 Inst. de Astrofísica de Andalucía (CSIC), 18080 Granada, Spain

16 Università di Padova and INFN, 35131 Padova, Italy

17 Università dell'Insubria, Como, 22100 Como, Italy

18 Unitat de Física de les Radiacions, Departament de Física, and CERES-IEEC, Universitat Autònoma de Barcelona, 08193 Bellaterra, Spain

19 Institut de Ciències de l'Espai (IEEC-CSIC), 08193 Bellaterra, Spain

20 Japanese MAGIC Consortium, Division of Physics and Astronomy, Kyoto University, 606-8501 Kyoto, Japan

${ }^{21}$ Finnish MAGIC Consortium, Tuorla Observatory, University of Turku and Department of Physics, University of Oulu, 900147 Oulu, Finland

22 Inst. for Nucl. Research and Nucl. Energy, 1784 Sofia, Bulgaria

23 Universitat de Barcelona, ICC, IEEC-UB, 08028 Barcelona, Spain

24 Università di Pisa, and INFN Pisa, 56126 Pisa, Italy

25 Now at: NASA Goddard Space Flight Center, Greenbelt MD 20771; and Department of Physics and Department of Astronomy, University of Maryland, College Park MD 20742, USA

26 Now at: École polytechnique fédérale de Lausanne (EPFL), 1015 Lausanne, Switzerland
27 Now at: Department of Physics \& Astronomy, UC Riverside, CA 92521, USA

${ }_{28}$ Finnish Centre for Astronomy with ESO (FINCA), Turku, Finland

29 Also at: INAF via Tiepolo Giambattista, 34131 Trieste, Italy

30 Also at Instituto de Fisica Teorica, UAM/CSIC, 28049 Madrid, Spain

31 Now at: Stockholm University, Oskar Klein Centre for Cosmoparticle Physics, 10691 Stockholm, Sweden

32 Now at: GRAPPA Institute, University of Amsterdam, 1098 XH Amsterdam, The Netherlands

33 Kavli Institute for Particle Astrophysics and Cosmology, SLAC National Accelerator Laboratory, Stanford University, Stanford, CA 94305, USA

34 Cahill Center for Astronomy \& Astrophysics, California Institute of Technology, 1200 E California Blvd, Pasadena, CA 91125, USA

35 Department of Physics, Purdue University, 525 Northwestern Ave, West Lafayette, IN 47907, USA

36 Aalto University Metsähovi Radio Observatory, Metsähovintie 114, 02540 Kylmälä, Finland

37 Aalto University, Department of Radio Science and Engineering, 02150 Espoo, Finland

38 Department of Physics, University of Crete, Greece 


\section{Appendix A: Detailed Swift-UVOT and XRT results}

Table A.1. Summary of Swift observations on PKS 1424+240 in 2009-2010 with the two instruments, XRT and UVOT.

\begin{tabular}{lccccccc}
\hline \hline Obs. ID & Date & $\begin{array}{c}\text { Start time } \\
{[\mathrm{MJD}]}\end{array}$ & $\begin{array}{c}\text { XRT expos. } \\
\text { (PC-mode) [s] }\end{array}$ & $\begin{array}{c}\text { XRT counts } \\
{\left[\mathrm{cnts} \mathrm{s}^{-1}\right]}\end{array}$ & $\begin{array}{c}\text { Pile-up } \\
\text { correction }\end{array}$ & $\begin{array}{c}\text { UVOT exposure } \\
{[\mathrm{s}]}\end{array}$ & Filters \\
\hline 38104001 & 11-Jun.-2009 & 54993.1840 & 1732 & 0.43 & $\mathrm{y}$ & 1707 & all \\
38104002 & 12-Jun.-2009 & 54994.1181 & 1785 & 0.49 & $\mathrm{y}$ & 1736 & all \\
38104003 & 13-Jun.-2009 & 54995.1965 & 2263 & 0.45 & $\mathrm{y}$ & 2215 & all \\
38104004 & 14-Jun.-2009 & 54996.0674 & 2311 & 0.5 & $\mathrm{y}$ & 2261 & all \\
38104005 & 15-Jun.-2009 & 54997.1333 & 2093 & 0.89 & $\mathrm{y}$ & 2044 & all \\
38104006 & 16-Jun.-2009 & 54998.2153 & 1132 & 0.88 & $\mathrm{y}$ & 1106 & all \\
38104007 & 17-Jun.-2009 & 54999.1570 & 1269 & 0.84 & $\mathrm{y}$ & 1220 & all \\
38104008 & 18-Jun.-2009 & 55000.2132 & 1132 & 0.45 & $\mathrm{y}$ & 1110 & all \\
38104009 & 19-Jun.-2009 & 55001.2208 & 1132 & 0.52 & $\mathrm{y}$ & 1108 & all \\
38104010 & 20-Jun.-2009 & 55002.1695 & 1403 & 0.27 & $\mathrm{n}$ & 1356 & all \\
39182001 & 24-Nov.-2009 & 55159.3083 & 4696 & 0.14 & $\mathrm{n}$ & 4691 & UW1 \\
39182002 & 22-Jan.-2010 & 55218.0097 & 1005 & 0.09 & $\mathrm{n}$ & 984 & all \\
39182003 & 22-Jan.-2010 & 55218.0764 & 1476 & 0.09 & $\mathrm{n}$ & 1477 & UM2 \\
40847001 & 21-Nov.-2010 & 55521.7549 & 1946 & 0.56 & $\mathrm{n}$ & 1900 & BUW1M2W2 \\
41539001 & 29-Nov.-2010 & 55529.5202 & 1131 & 0.47 & $\mathrm{n}$ & 1105 & all \\
\hline
\end{tabular}

Table A.2. Results of the analysis on Swift -XRT data.

\begin{tabular}{|c|c|c|c|c|c|c|c|}
\hline Obs. ID & $\begin{array}{l}\text { Date } \\
{[\mathrm{MJD}]}\end{array}$ & $\begin{array}{c}\text { Exposure } \\
{[\mathrm{s}]}\end{array}$ & $\begin{array}{l}\text { Flux } 0.5-10 \mathrm{keV} \\
{\left[10^{12} \mathrm{erg} \mathrm{cm}^{-2} \mathrm{~s}^{-1}\right]}\end{array}$ & Flux err & Index & Idx err & Red. $\chi^{2} / \mathrm{NDF}$ \\
\hline 38104001 & 54993.1840 & 1732 & - & - & - & - & - \\
\hline 38104002 & 54994.1181 & 1785 & 22.7 & 1.1 & 2.54 & 0.09 & $1.23 / 25$ \\
\hline 38104003 & 54995.1965 & 2263 & 26.7 & 1.1 & 2.55 & 0.09 & $1.00 / 28$ \\
\hline 38104004 & 54996.0674 & 2311 & 23.1 & 1.1 & 2.55 & 0.09 & $1.33 / 30$ \\
\hline 38104005 & 54997.1333 & 2093 & 47.3 & 1.5 & 2.37 & 0.06 & $1.03 / 52$ \\
\hline 38104006 & 54998.2153 & 1132 & 43.9 & 2.1 & 2.41 & 0.10 & $1.08 / 28$ \\
\hline 38104007 & 54999.1570 & 1269 & 41.3 & 1.0 & 2.42 & 0.09 & $1.15 / 30$ \\
\hline 38104008 & 55000.2132 & 1132 & 29.8 & 1.8 & 2.55 & 0.14 & $1.58 / 13$ \\
\hline 38104009 & 55001.2208 & 1132 & 26.2 & 1.9 & 2.34 & 0.16 & $1.7 / 15$ \\
\hline 38104010 & 55002.1695 & 1403 & - & - & - & - & - \\
\hline 39182001 & 55159.3083 & 4696 & 6.1 & 0.4 & 2.53 & 0.09 & $1.06 / 31$ \\
\hline 39182002 & 55218.0097 & 1005 & - & - & - & - & - \\
\hline 39182003 & 55218.0764 & 1476 & - & - & - & - & - \\
\hline 40847001 & 55521.7549 & 1946 & 17.3 & 0.9 & 2.34 & 0.08 & $1.40 / 33$ \\
\hline 41539001 & 55529.5202 & 1131 & 13.2 & 1.1 & 2.36 & 0.14 & $1.23 / 15$ \\
\hline
\end{tabular}

Notes. The flux and spectral indices have been derived with a fit on XRT data in the range $0.5-10 \mathrm{keV}$ using a simple power-law model with photo-electric absorption (WABS model in XSPEC for $n_{\mathrm{H}}=3.1 \times 10^{20} \mathrm{~cm}^{-2}$ ). 


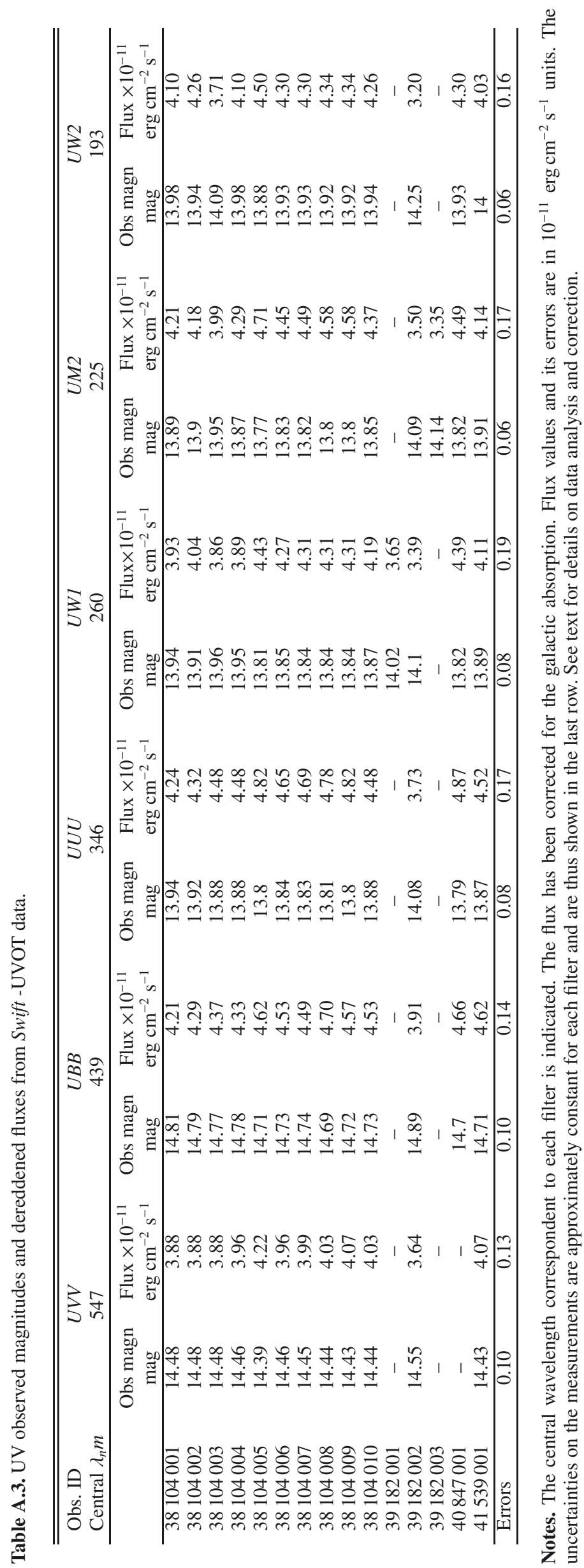

